\title{
Stable Homology as an Indicator of Manifoldlikeness in Causal Set Theory
}

\author{
Seth Major ${ }^{1}$, David Rideout ${ }^{2}$, and Sumati Surya ${ }^{3}$ \\ ${ }^{1}$ Hamilton College, Clinton, NY, USA \\ ${ }^{2}$ Perimeter Institute for Theoretical Physics, Waterloo, ON, Canada \\ ${ }^{3}$ Raman Research Institute, Bangalore, India
}

October 30, 2018

\begin{abstract}
We present a computational tool that can be used to obtain the "spatial" homology groups of a causal set. Localisation in the causal set is seeded by an inextendible antichain, which is the analog of a spacelike hypersurface, and a one parameter family of nerve simplicial complexes is constructed by "thickening" this antichain. The associated homology groups can then be calculated using existing homology software, and their behaviour studied as a function of the thickening parameter. Earlier analytical work showed that for an inextendible antichain in a causal set which can be approximated by a globally hyperbolic spacetime region, there is a one parameter sub-family of these simplicial complexes which are homological to the continuum, provided the antichain satisfies certain conditions. Using causal sets that are approximated by a set of $2 \mathrm{~d}$ spacetimes our numerical analysis suggests that these conditions are generically satisfied by inextendible antichains. In both $2 \mathrm{~d}$ and $3 \mathrm{~d}$ simulations, as the thickening parameter is increased, the continuum homology groups tend to appear as the first region in which the homology is constant, or "stable" above the discreteness scale. Below this scale, the homology groups fluctuate rapidly as a function of the thickening parameter. This provides a necessary though not sufficient criterion to test for manifoldlikeness of a causal set.
\end{abstract}

\section{Introduction}

Any approach to quantum gravity which assumes an underlying Plank scale spacetime discreteness also requires, alongside, a description of how continuum topology 
and geometry arise from the discrete substructure. Since the continuum description is apparently robust down to sub-nuclear scales, the effects of discreteness must be hidden from standard physics, though the possibility that its signatures can leak out to scales accessible to current observations and experiments has not been ruled out. Replacing spacetime with a collection of discrete elements cannot suffice, since the only natural topology is the discrete one, and the only natural geometry is one which makes an element infinitely far (or close) to every other element. From purely continuum considerations one is then led to conclude that the discrete elements must, at a minimum, be supported by additional relations.

For a theory based on Lorentzian spacetimes, the most natural relation is the causal relation. The importance of the causal relation to continuum Lorentzian geometry is emphasized by Malament's result that the causal structure determines the conformal class for strongly causal spacetimes [1]. The conformal factor is the remaining degree of freedom, and is determined by the local volume element. The causal set theory (CST) approach to quantum gravity gives primacy to the causal relations while assuming a fundamental discreteness. The discrete substructure that replaces spacetime is taken to be a locally finite partially ordered set or causal set, where the order relation maps to the causal relation in the continuum approximation of the theory [2, 3, 4].

In CST, the approach to the continuum has a concrete prescription in terms of a faithful embedding $\Phi: C \rightarrow(M, g)$ from a causal set $C$ to a spacetime $(M, g)$. If such a $\Phi$ exists, then $C$ is said to be approximated by $(M, g)$. $\Phi$ is an order preserving map which preserves, on average, the local correspondence between cardinality and spacetime volume. By this we mean that the events in $M$ to which the elements of $C$ are mapped arise from a Poisson process at some given density $V_{c}^{-1}$. Thus, the average number of causal set elements in a given spacetime volume $V$ is given by $\langle N\rangle=V / V_{c}$. Putting this together with the Malament result, one obtains, in the continuum approximation of CST, the maxim "Order + Number $\sim$ Spacetime". This construction allows several kinematical questions to be addressed within CST without explicit reference to the dynamic 1 .

A valuable feature of the random lattice associated with the continuum approximation is that it also implies local Lorentz invariance [9]. The randomness however makes the task of extracting spacetime topology and geometry from the causal set more difficult than for a regular lattice in which each element has a fixed finite valency. On the other hand, for a causal set that is approximated by Minkowski spacetime, the elements have infinite valency, a legacy of local Lorentz invariance. This means that there is no useful (local) definition of nearest neighbours for a given

\footnotetext{
${ }^{1}$ A dynamical law may be expressed with CST, for example, as a (quantum) measure on the space of histories, e.g. as arising from a process of sequential growth [5, 6], or in terms of an action functional on causal sets [7, 8].
} 
element in such a causal set. However, the prescription for a faithful embedding is itself concrete enough that substantial progress has been made in extracting continuum information from the causal set. In particular, one now has a reasonable understanding of how spacetime dimension [10], time-like and spacelike distances [11, 12], and a localised Ricci scalar [7] can be constructed intrinsically in a causal set.

The construction of spacetime homology has been addressed in detail in [13], and the current work is a numerical follow-up of the analytical results. Rather than consider the full spacetime topology, this construction uses a spatial localisation of the causal set to extract continuum information. In any causal set $C$, a set of mutually unrelated elements forms an antichain, and a complete set of such elements forms an inextendible antichain $A$. This is the analog of a "fixed time slice" and can be used to obtain a frame-dependant localisation. Since the elements of $A$ are unrelated (or spacelike related in the continuum approximation) it has insufficient intrinsic topological information. Instead $A$ can be enriched by borrowing information from its embedding in the causal set. One way to do this is to "thicken" it to some $T(A)$ by including elements that are in its neighbourhood. This thickened antichain $T(A)$ is endowed with a richer topological and geometric structure compared to $A$, and the idea is to use it to compare to the continuum.

In [14, 13] spacetime volume $n$ was used as a thickening parameter. For an $A$ which admits a certain "separation of scales" it was shown that there is a range of $n$ for which the nerve simplicial complex constructed from $T_{n}(A)$ is homological to the continuum with high probability. As discussed in greater detail in Section 2 this is possible so long as there exists an $n$ such that $T_{n}(A)$ is "thick enough" locally compared to the discreteness scale, but also thin enough to admit a sensible localisation. This separation of scales is guaranteed in the continuum limit with the discreteness scale going to zero as the sprinkling density is made larger. However, given a fixed sprinkling density, it is not obvious how generic such antichains are. Thus it becomes pertinent to ask, for a causal set $C$ which is approximated by a spacetime, how likely is it that one will pick an inextendible $A$ in $C$ which admits this separation of scales. The current work addresses this question using numerical techniques. Using causal sets obtained from discretisations of $2 \mathrm{~d}$ and $3 \mathrm{~d}$ spacetimes, as well as those generated by other means, we propose a necessary but not sufficient criterion for manifoldlikeness of a causal set.

We use a Cactus based causal set computational tool [15, 16] to construct causal sets, pick out a thickened antichain and construct its nerve simplicial complex. Subsequently, we employ the CHomP homology package [17] to calculate the spatial homology groups for a suite of causal sets. The first class of causal sets arise from discretisations of the following spacetime regions: (i) the flat $2 \mathrm{~d}$ and $3 \mathrm{~d}$ intervals with topologies $I \times I$ and $I \times I \times I$ respectively, (ii) the 2 d cylinder spacetime with 
topology $S^{1} \times I$, (iii) 3d flat spacetimes with topologies $T^{2} \times I$ and $S^{1} \times I \times I$, with different spatial sizes to examine Kaluza-Klein type effects, (iv) the "split" $2 \mathrm{~d}$ trousers, $I \sqcup I \rightarrow I$ and (v) the $2 \mathrm{~d}$ expanding FRW spacetime with topology $S^{1} \times I$. The second class of causal sets arise from non-manifoldlike considerations: (i) from the transitive percolation dynamics which belongs to the classical sequential growth class of dynamics studied in [5, 18] and (ii) the tower-of-crowns poset which is a "crystalline" causal set obtained via a regular discretisation of the $2 \mathrm{~d}$ cylinder spacetime.

Our results demonstrate that, for causal sets that are approximated by a class of $2 \mathrm{~d}$ or $3 \mathrm{~d}$ spacetimes, the continuum homology groups are stable (constant) over a large range of thicknesses. While some of the $3 \mathrm{~d}$ computations, being more CPU intensive, give only an indication of stability, the $2 \mathrm{~d}$ computations allow us to identity quantitative correlations between the causal set and the continuum. In the $2 \mathrm{~d}$ case, for each causal set obtained from a discretisation of a spacetime from the above set, we use an ensemble of 100-200 randomly generated antichains and obtain homology as a function of thickening for each. We find that the continuum homology has a tendency to appear in a contiguous range of thicknesses. We characterise the stability of the homology in terms of a dimensionless parameter, the "stability ratio" $s_{R}$ (see eqn (3) and the following discussion) and define a region to be stable only if $s_{R} \gtrsim 1$. We find that the continuum homology does not appear as the most stable region. However, when the width of the stable region is required to be much larger than the discreteness scale, the continuum homology appears as the first stable region for a high percentage of the trials. Moreover, the homology changes rapidly before the first stable region begins, and when comparing across different trials is uncorrelated for small $n$.

While our 3d results support these conclusions, the quantitative analysis is limited by computational constraints. In particular, only minimal antichains are used in all the computations. Such an antichain, made up of the minimal elements of the causal set, is extrinsically as flat as possible, since it stays close to the flat boundary of the sprinkling region. For this choice, a stable region with the continuum homology starts to appear, but the computation cannot always be carried out for large enough thicknesses to establish whether the region is stable. An interesting class of causal sets are those obtained from discretisations of Kaluza Klein type spacetimes, with topologies $T^{2} \times I$ and $S^{1} \times I \times I$, where the size of the compactified (internal) dimension $S^{1}$ with respect to the non-compact (external) spacetime $S^{1} \times I$ or $I \times I$ is varied. When the compactification scale is small, of order of the sprinkling scale, we find that its topology does not show up in the stable homology which matches only that of the external spacetime. Thus, one gets an effective $2 d$ topology as expected. As the size of the compactified region is increased, an intermediate stable homology begins to arise which suggests the presence of an internal manifold. 
Apart from validating the analytic results of [13] our results suggest a test for manifoldlikeness in a causal set 2 Namely, for a causal set $C$, if in a statistically large sample of randomly chosen antichains in $C$ (i) the homology groups for the first stable region obtained from each antichain agree for most of the trials and (ii) for each of the sampled antichains at thickness of the order of the discreteness scale the homology changes rapidly but does not agree over even a small fraction of the trials, then $C$ satisfies a necessary but not sufficient condition for manifoldlikeness. We demonstrate our test on causal sets generated via a transitive percolation dynamics [5. 18] as well as for the tower of crowns causal sets. We find that while causal sets generated by a class of transitive percolation dynamics qualitatively suggest manifoldlikeness, they do not pass the quantitative tests. The tower-of-crowns causal set, which we examine analytically, is an example of a non-manifoldlike causal set in which there is a consistent first stable homology region, but for a large "preferred" set of antichains it begins immediately, i.e., there is no initial region of varying homology.

Our results and analysis suggest that the criterion put forth may be more generally valid. It would be useful to carry out the computations in higher dimensions as a check, but as the spacetime dimensions increase, so does the dimension of an average simplex in the nerve. While the Cactus based programs are very efficient in generating the nerves for a large range of thicknesses for causal sets with $O\left(10^{5}\right)$ elements, the homology program CHomP baulks (and reasonably so) at these very large simplices.

We devote the next section to preliminaries, and describe the computational set up in Section 2.2. We present the stable homology results in Section 3 and the tests for manifoldlikeness in Section 4. In section 5 we discuss our results and some open questions.

\section{Preliminaries}

A causal set (or causet) $C$ is a finite or countable collection of elements, along with a binary order relation $\prec$ which is transitive $(x \prec y$ and $y \prec z \Rightarrow x \prec z$, irreflexive $(x \nprec x)$, and locally finite $(|\operatorname{past}(x) \cap \operatorname{fut}(y)|<\infty$. Here, $\operatorname{past}(x) \equiv\{y \in C \mid y \prec x\}$, fut $(x) \equiv\{y \in C \mid x \prec y\}$.

A subset $S$ of a causal set $C$ is implicitly endowed with the causal relation of $C$ restricted to the subset $S$, and is referred to as a sub-causal set or subcauset with $S \subseteq C$. The future and past of an element $x \in S$ restricted to $S$ are denoted

\footnotetext{
${ }^{2}$ See 21 for a different test for manifoldlikeness for causal sets that are approximated by an interval in $2 \mathrm{~d}$ Minkowski spacetime.
} 
as fut $(x, S)$ and past $(x, S)$, respectively. A chain is a subcauset which is totally ordered so that every pair of elements is related, and an inextendible antichain $A$ is a subcauset of the causal set $C$ which has no relations among its elements, and for which every element $x \in C$ is related to at least one element of $A$. A maximal element $x \in S \subseteq C$ has $\operatorname{fut}(x, S)=\emptyset$ and a minimal element $y \in S \subseteq C$ has $\operatorname{past}(y, S)=\emptyset$.

Associated with $A$ is its "volume thickened" neighbourhood, the subcauset

$$
T_{n}(A)=\{x \in(\operatorname{fut}(A) \cup A)|| \operatorname{past}(x) \backslash \operatorname{past}(A) \mid \leq n\},
$$

where $n$ is any non-negative integer, and $T_{0}(A) \equiv A 3$ In [13] $T_{n}(A)$ was used to prove a correspondence between the continuum and the causal set homology and hence will play a crucial role in our analysis. The parameter $n$ corresponds to a scale and can be used to compare with continuum expectations.

Apart from its use in homology studies, this thickening also has the rather interesting property that it leads to an eventual smoothing out of the original antichain, i.e., the extrinsic curvature is gradually "uniformised" on the antichain obtained from the maximal elements of the thickening as shown in Fig 迎. The reason for this is that the volume thickening tends to "fill up" the valleys, or regions of negative extrinsic curvature regions faster than it grows the hills, or regions of positive extrinsic curvature5. A past volume thickening has the opposite effect and uniformises extrinsic curvature to the past. A qualitative understanding of this smoothing property in the continuum comes from comparing the past volumes down to $\Sigma$, of two elements $p, q$ in the future of a spacelike hypersurface $\Sigma$, both a fixed proper time from $\Sigma$. If $v(p) \equiv I^{-}(p) \cap \Sigma$ contains a positive extrinsic curvature region and $v(q) \equiv I^{-}(q) \cap \Sigma$ a negative extrinsic curvature region (where $I^{ \pm}(x)$ denotes the causal future and past of $x$ ) then $v(p)>v(q)$. Thus $q$ will be incorporated into a smaller volume thickening of $\Sigma$ than $p$, which suggests that the negative extrinsic curvature regions thicken "faster" than the positive curvature regions.

\subsection{Constructing the Nerve Simplicial Complex}

Starting from a volume thickening $T_{n}(A)$ of an antichain, we begin by constructing "shadows" of the pasts of the maximal elements $\left\{m_{i}\right\}$ of $T_{n}(A)$ onto $A$

$$
S_{n}^{i} \equiv\left(m_{i} \cup \operatorname{past}\left(m_{i}\right)\right) \cap A .
$$

\footnotetext{
${ }^{3}$ This differs marginally from the definition used in 13 , where the counting included $x$.

${ }^{4}$ In several of the figures including this one, we have "thinned" the embeddings in order to have visual clarity in a black and white printout. However, they are best viewed in colour.

${ }^{5}$ This smoothing out of extrinsic curvature appears to be at least qualitatively not-unlike a Ricci-type flow although it is difficult to construct a local flow equation to make a clear comparison 22 .
} 


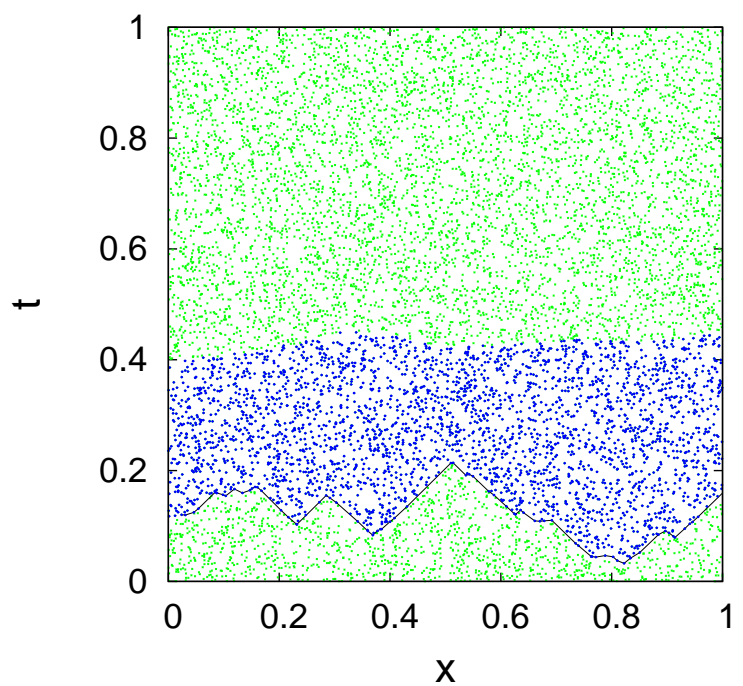

Figure 1: A causal set obtained from a sprinkling of $N=20,000$ elements into a portion of the 2 d cylinder spacetime $S^{1} \times I$, with $x=0 \sim x=1$. In order to avoid cluttering, the relations have not been drawn, but are determined by the $45 \mathrm{deg}$ light cones. A random inextendible antichain has non-uniform induced extrinsic curvature (emphasised by the jagged line). Thickening it to a volume of $n=1000$ tends to uniformise the curvature.

The collection $\mathcal{O}_{n}=\left\{S_{n}^{i}\right\}$ covers $A$ since $\cup_{i} S_{n}^{i}=A$ which is also locally finite. The associated nerve simplicial complex $\mathcal{N}\left(\mathcal{O}_{n}\right)$ is constructed by assigning to every element of $\mathcal{O}_{n}$ a vertex, and to every $n$-wise intersection, an $n-1$-simplex [23].

Fig 2 gives an example of two different nerves constructed from two different locally finite coverings of the circle. These can be thought to arise from a continuum

(a)

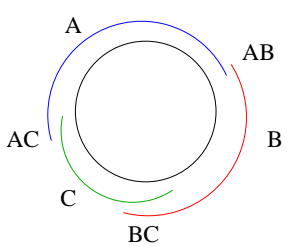

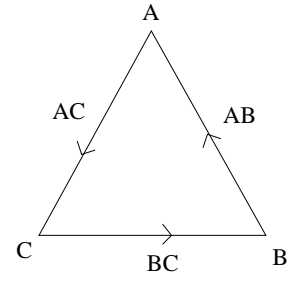

(b)

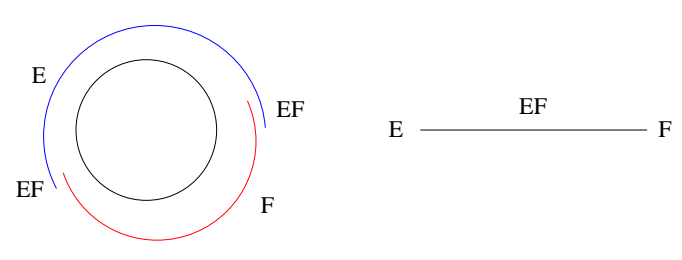

Figure 2: Nerves constructed from two different coverings of $S^{1}$. (a) This nerve is homological to the circle since $H_{0}=\mathbb{Z}, H_{1}=\mathbb{Z}$. (b) This nerve is homological to a point since $H_{0}=\mathbb{Z}$ is the only non-trivial homology group.

version of the shadow construction for a simple spacetime like the flat cylinder $S^{1} \times \mathbb{R}$, where $d s^{2}=-d t^{2}+d x^{2}$, and $x=0 \sim x=1$. Starting with the $t=0$ slice, we can volume thicken to the future. As shown in 13 the future boundary of such a thickening is itself homeomorphic to $S^{1}$, and one can pick a few points on this to 
obtain a finite shadow cover of the $t=0$ slice. For small thickness, it is possible to stay within the so called convexity volume $v_{c}$ associated with the slice, which is the largest thickening for which the shadows are convex subsets of $S^{1}$. For such finite coverings, a theorem due to De Rham and Weil tells us that the nerve simplex is homotopic to $S^{1}$ [24]. However, once the convexity volume is passed, the simplicial complexes are not necessarily homotopic to $S^{1}$. A useful example of how higher dimensional homology can arise from such a construction is given in Fig 3 ,

(a)
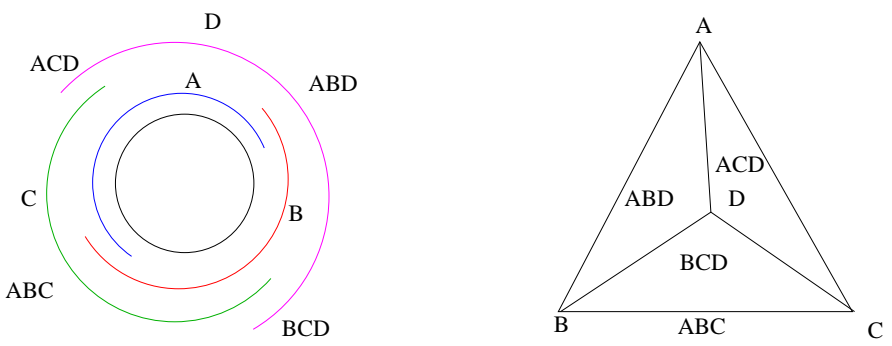

Figure 3: A covering of $S^{1}$ made up of 4 non-convex subsets. The three way intersections are all non-zero, resulting in 4 2-simplexes which make up the boundary of a tetrahedron. However, there is no 4-way intersection, so the interior of the tetrahedron is left empty. Thus, the non-trivial homology groups are $H_{0}=\mathbb{Z}$ and $H_{2}=\mathbb{Z}$, even though the covering is of a 1-dimensional manifold.

Past the convexity volume is another important regime associated with the "cosmological scale" $v_{\lambda}$. This is the thickening at which at least one of the shadows $S_{c}$ encompasses all of the initial hypersurface. Such a scale can be infinite as in the case of Minkowski spacetime, but is finite for compact spacetime regions. Not only does $S_{c}$ intersect all other shadows, but also all their intersections. This means that if the nerve contains a non-trivial cycle without the vertex $S_{c}$, its addition must necessarily collapse it to a trivial cycle. Hence the existence of such an $S_{c}$ "washes" out non-trivial homological information contained in the spatial slice. This cosmological scale is therefore the thickening limit beyond which useful spatial homological information cannot be extracted.

In [13] it was shown that, for a causal set that is approximated by a globally hyperbolic region of spacetime at a given sprinkling density $V_{c}^{-1}$, the nerve $\mathcal{N}\left(\mathcal{O}_{n}\right)$ is homological to the continuum for a large range of $n$ 's, as long as $A$ satisfies a certain separation of scales. In analogy with the continuum, $n$ must be less than the convexity number $n_{c} \equiv v_{c} / V_{c}$. Moreover, not only should $n \gg 1$, i.e., be far from the discreteness scale $(n \sim 1)$, but also be large enough that $T_{n}(A)$ is not too "thin" in patches; since the thickening is uneven if $A$ has a non-uniform extrinsic curvature to start with, the maximal elements of $T_{n}(A)$ may continue to lie close to $A$ even for $n \gg 1$. This determines an additional scale $n_{0}$, related to a minimal proper distance. The separation of scales requirement is then $1 \ll n_{0} \ll n \ll n_{c}$, 
where the $\ll$ are required to take into account the possibility of large fluctuations. For example, the volume containing $n_{c}$ sprinkled elements can be much larger than the convexity volume $v_{c}$ beyond which the continuum results are not valid. The separation of scales therefore allows the possibility of a large range of $n$ for which $\mathcal{N}\left(\mathcal{O}_{n}\right)$ is homological to the continuum. Fig (4) shows how the separation of scales manifests itself in the $2 \mathrm{~d}$ flat cylinder spacetime with topology $S^{1} \times I$.

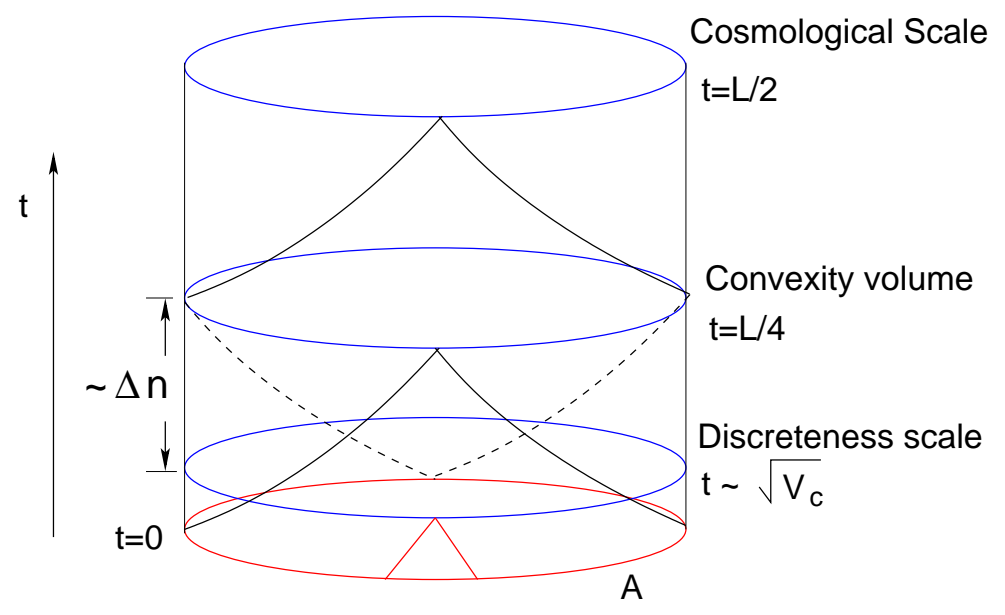

Figure 4: The convexity volume and the cosmological scale for a $t=0$ slice in the $2 \mathrm{~d}$ cylinder spacetime with spatial size $L$ are shown. For a causal set $C$ which is approximated by this region of the spacetime, the minimal inextendible antichain $A$ in $C$ is approximated by the $t=0$ slice. While the region of first stable homology for the continuum slice is $t \in(0, L / 4)$, that for $A \subset C$ lies in the region $t \in\left(\gtrsim \sqrt{V_{c}}, L / 4\right)$ where $\sqrt{V_{c}}$ represents, in this case, the discreteness timescale.

If it can be shown that inextendible antichains in such a causal set $C$ generically satisfy the separation of scales, then the appearance of a stable homology can conversely be taken to be an indicator of manifoldlikeness. The analytical results, while robust, do not help address this question.

\subsection{The Computations}

Our primary aim in this work is to further the idea that stable homology can be used to test for manifoldlikeness of a causal set. Given the complexity of causal sets, such a test requires the use of numerical tools described below.

The construction of the nerve simplicial complex uses a causal sets toolkit [16] within the Cactus high performance computing framework [15. The toolkit consists of a number of modules called "thorns", which provide various functionalities needed to perform computations with causal sets. The first step is to choose a spacetime 
$(M, g)$ from which a causal set $C$ can be obtained. Next, we sprinkle into the spacetime, which yields a number of causet elements sampled from a Poisson distribution with mean $N$. (It is to be understood below that phrases such as "a sprinkling of $N=20,000$ elements" means we sprinkle with $N$ set to 20,000 , not that the causal set necessarily contains 20,000 elements.) The induced causal relations between the elements are then computed to obtain $C$. This latter process requires a detailed knowledge of the causal structure of $(M, g)$ which is not always readily available. Therefore the suite of spacetimes used is currently limited to a set whose causal structure is explicitly known. The software provides for sprinkling into a variety of topologies of conformally flat spacetime, for any spacetime dimension up to $8+1$. It also provides many fundamental set operations which are needed for the computations described in this paper, such as computing pasts and futures, unions and intersections, and minimal and maximal elements.

Of course, one also wants to generate causal sets in other ways besides sprinkling into spacetimes, since this avoids an a priori assumption of manifoldlikeness. The causal sets toolkit provides several examples of such causal sets, some of which are generated via so-called sequential growth dynamics [5].

Once a causal set $C$ has been generated, an inextendible antichain $A$ is picked in one of two ways. The simplest choice is the set of minimal elements. This ties $A$ to the choice of the arbitrarily defined bounding frame for the sprinkling, and is therefore unnatural. A more natural and robust procedure is to choose a randomly generated inextendible antichain, and to subsequently consider a large number of such choices, to account for statistical fluctuations.

Since these random antichains are used in a crucial way in the $2 \mathrm{~d}$ computations, we discuss it in some detail. We use the following algorithm for selecting an inextendible antichain. First, select an element from the causal set at random with a uniform distribution, but restrict this first selected element of the antichain to have label $\leq M / 2$, where the labels of the elements are in $[0, \ldots, M-1]$ ( $M$ is the total number of elements in the causal set). The labels of the elements are given in the order of their time coordinates for sprinklings, or as described in Section 4 for non-sprinkled causal sets. The labeling is always a natural labeling, meaning that if $i \prec j$ in the causet order then their labels satisfy $i<j$. Thus this restriction has the effect of causing the antichain to tend to live in the lower half of the causal set, which is useful because we always thicken it to the future.

Next, select a second element which is unrelated to the first, again with a uniform distribution on the eligible elements (i.e. the set of elements unrelated to the first, irrespective of their labels). Then select a third which is unrelated to the first two, again with a uniform distribution. Repeat until there are no elements remaining which are unrelated to any element selected thus far. This will select an inextendible antichain at random, from any causal set. Fig 5 depicts a collection of such random 
antichains. Hugging the $t=0$ surface is the minimal antichain. The figure indicates

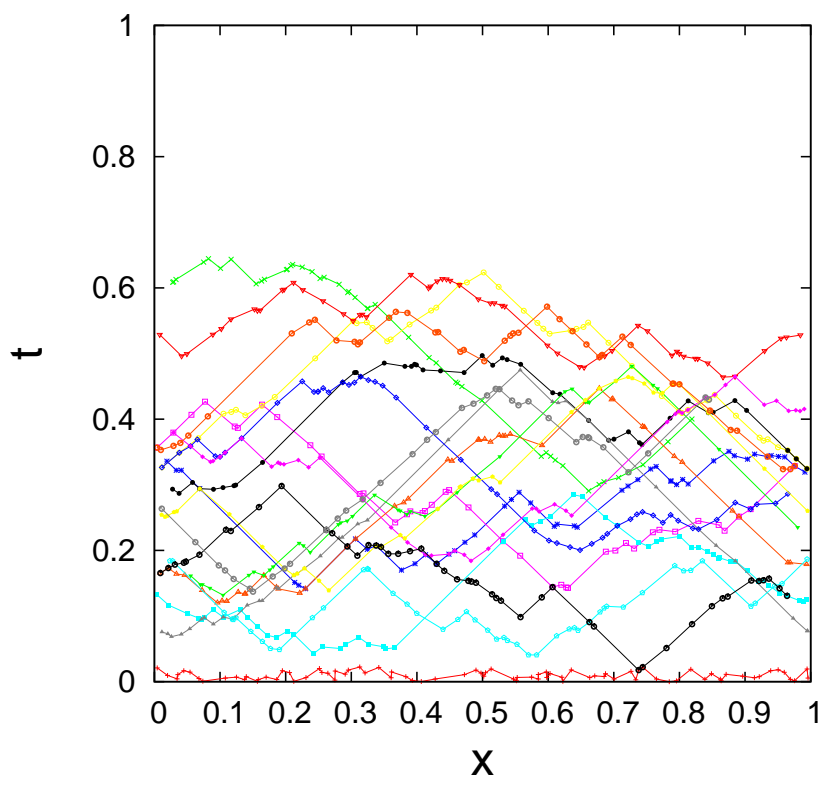

Figure 5: A selection of randomly generated inextendible antichains in a causal set obtained from a sprinkling of $N=5,000$ elements into the 2 d cylinder spacetime $S^{1} \times I$, with $x=0 \sim x=1$. The minimal antichain (not randomly generated) hugs the $t=0$ line. We have used lines to connect the elements of the individual antichains for visual clarity.

that the algorithm tends to pick out antichains with highly varying induced extrinsic curvature, and hence those that are "almost null" with respect to the preferred slicing of the spacetime. These antichains seem to be the least likely to satisfy the analytical criterion for separation of scales, since "thin" patches may persist up to the convexity volume scale. In the case of an embedding into the cylinder spacetime $d s^{2}=-d t^{2}+d x^{2}$, with $x=0 \sim x=1$ and topology $S^{1} \times I$, let us consider the extreme example of a two-element antichain for which the nerve construction is inadequate; the only two possible homologies are $H_{0}=\mathbb{Z}^{2}$ or $H_{0}=\mathbb{Z}$, both with $H_{1}=0$.

We use this example to argue that the probability distribution on inextendible antichains imposed by the above algorithm is far from uniform, and in fact tends to favor more 'pathological' antichains, which have fewer elements and wider 'almost null' segments. To see this, for an arbitrary antichain $A$, we define a quantity $n_{A}(x)$ to be the number of inextendible antichains which include all of $A$ and $x$ as well. For $A$ equal to a single element $x$ in $S^{1} \times \mathbb{R}$ spacetime, one expects to find a $y$ such that $n_{A}(y)=1$. If we wanted to select each inextendible antichain with equal probability, then, if $x$ were the first element of our antichain, then we would have to weight $y$ with a much smaller probability than some other element $z$ for which $n_{A}(z)$ » 
1. However, the above algorithm does not. This suggests that the algorithm is weighted toward smaller inextendible antichains, which contain numerous almostnull segments, and intuitively are expected to have a larger extrinsic curvature. It is therefore all the more challenging to test the homology construction on these antichains. It is in this sense that we claim that our results are generic.

Having chosen the antichain, the toolkit contains a module which constructs the thickened antichain. As discussed earlier, the thickening $T_{n}(A)$ of a randomly chosen $A$ tends to uniformise the extrinsic curvature of antichain $A_{1}^{\prime}$ constructed from maximal elements of $T_{n}(A)$ for large enough $n$. The antichain $A_{1}^{\prime}$ can then be completed using the uniform distribution as described above to obtain an inextendible $A_{1}$, from which a new set of thicknesses $T_{n}\left(A_{1}\right)$ can be constructed. The randomness associated with $A$ and its associated non-uniform extrinsic curvature is thus at least partially tamed. While this procedure appears promising, it is not a a priori obvious how large $n$ needs to be to uniformise a randomly chosen antichain. In order to avoid introducing further arbitrary parameters, we make do with the first thickening $T_{n}(A)$.

Next, having picked a large enough thickness $n_{m}$, the causal sets toolkit constructs a nerve simplicial complex for each $T_{n}(A), n \in\left[0, n_{m}\right]$, and stores it in a format that can be accessed by the CHomP computational homology package [17. The core CHomP engines use a variety of cubical homology algorithms to compute homology groups. We use the "homsimpl" program, which acts as a front end to CHomP for abstract simplicial complexes. Before doing so, we simplify the nerve simplicial complex with a Perl program developed by Pawel Pilarczyk which removes redundant vertices. This reduces the average size of the simplices and thus improves the computational efficiency substantially. In performing a large number of trials for a given causal set, finding their constant homology regions and assessing their stability, we made extensive use of bash programming.

In the computations we have to decide a priori what the maximum thickness $n_{m}$ should be. It suffices to thicken to the cosmological scale since we know that the homology is always trivial past this scale. Thus, $n_{m}$ must be chosen to be larger than all the possible cosmological scales. This can be done by generating a large sample of inextendible antichains in pretrials and finding their cosmological scales. In many of the trials the thickening is stopped only at or very close to the cosmological scale. However, as the sprinkling density increases, it becomes more computationally intensive to reach the cosmological scale. On the other hand, the cosmological scale is only a weak upper bound for the convexity volume. Since we are interested in establishing the existence of stable regions and showing that the first stable region is correlated to the continuum homology, it is then enough to thicken the antichain to some $n_{m}$ which is large enough to establish a first stable region. This is indeed what we do for the high density sprinklings. 
The computation of homology groups was by far the bottleneck in all our computations. As an illustration, consider an $N=5793$ element causal set obtained from a sprinkling into the flat $T^{2} \times I$ spacetime. For a 'typical' $T_{n}(A)$, with $n=45$, there are $5793^{2 / 3} \approx 323$ maximal elements in $T_{45}(A)$. Thus, approximately 323 sets cover the 323 or so elements in $A$. The nerve simplicial complex has 187 simplices, with dimensions as large as 19 or more (recall this means simply that there are 20 mutually overlapping sets in the cover). Since the number of subsimplices of a single 19-simplex alone is $2^{20}$, one can see that finding the homology groups of these complexes becomes a large task for any computer.

A qualitative picture begins to emerge from examining plots of homology versus thickness for the several examples considered. First, we notice that torsion coefficients are trivial in all examples and hence it suffices to consider the Betti numbers. This is undoubtedly a curiosity of our simulations; while we expect a constant homology region which reproduces the continuum homology, there are no known constraints on the homology outside of this region as long as it remains within the cosmological scales. It is possible that this is merely an artifact of our choice of torsion free spacetimes and the maximum dimensions we can handle. The plots generically show that as the thickening is increased from the discreteness scale to the cosmological scale, that there is an initial period of rapidly changing homology, followed by at least one region of constant homology, ending with the cosmological scale at which $H_{0}=\mathbb{Z}$ is the only non-trivial homology (see Fig 9 for example.).

In order to make a quantitative statement, we first need to define stability. Does it, for example, suffice for homology to be constant over 2 thicknesses, or 20 or 200 for us to deem it stable? We define the stability ratio to be

$$
s_{R} \equiv \frac{n_{\max }-n_{\min }}{n_{\min }}=\frac{\Delta n}{n_{\min }}
$$

where $n_{\min }, n_{\max }$ are the minimum and maximum thickness, respectively, for a contiguous region in which the homology is constant.

A natural definition of stability is to require that (a) $s_{R} \gtrsim \mathcal{O}(1)$ or $\Delta n \gtrsim \mathcal{O}\left(n_{\text {min }}\right)$, thus ruling out regions in which the homology is constant only in a relatively fleeting region $\Delta n \ll n_{\min }$. (For the purpose of the analysis presented here, we interpret the inequality $y \gtrsim \mathcal{O}\left(10^{x}\right)$ to mean $y \geq 5 \times 10^{x-1}$.) However, this does not exclude constant homology regions that are too close to the discreteness scale to reproduce continuum features, since for $n_{\text {min }} \sim \mathcal{O}(1)$, (a) is satisfied by a $\Delta n \sim \mathcal{O}(1)$. To avoid this, we need in addition a mesoscale $m_{s} \gg 1$ which is a lower limit below which continuum features are not expected, so that (b) $\Delta n \gtrsim \mathcal{O}\left(m_{s}\right)$ as well. A value $m_{s}=100$ is one of the lowest possible values that satisfies $m_{s} \gg 1$, and is natural as an order of magnitude estimate. That this choice is not restrictive is obvious, since it only sets the lower bound for $\Delta n$. Thus, we will call a region stable if (a) and (b) 
are satisfied with $m_{s}=100$, or more explicitly, that $\Delta n \geq 0.5 n_{\min }$ and $\Delta n \geq 50$.

From our simulations it is clear that the continuum homology does not typically appear as the region with the largest stability ratio. It does however appear as a stable region in a very large fraction of the trials. Moreover, in almost as many trials, it appears as the first stable region. This gives us a concrete hypothesis for manifoldlikeness, tested over hundreds of examples of causal sets obtained from $2 \mathrm{~d}$ as well as $3 \mathrm{~d}$ discretisations. Conversely, it provides a reliable method of obtaining the continuum homology if it exists (see Fig 9 for example).

In addition to stability, manifoldlikeness is also characterised by the existence of a rapidly varying homology for $n \sim \mathcal{O}(1)$. The most significant homology in this region is $H_{0}$, since at such thicknesses, connectivity has to be first established. In the $2 \mathrm{~d}$ examples, the number of disconnected components $k$, where $H_{0}=\mathbb{Z}^{k}$, rapidly decreases from large values to a steady small value, as $n$ increases from 1 to about 10. Moreover, when comparing across trials, one needs to check for initial (roughly up to $n=10$ ), rapidly changing homologies that are uncorrelated in different trials. This can be traced to the randomness of the discretisation more clearly apparent at small scales.

In the $3 \mathrm{~d}$ examples, computational constraints prevent us from performing a similar detailed analysis. In each case, we note that regions of relatively large stability corresponding to the continuum homology indeed do appear, but one is unable in all but a few cases to continue up to $n_{\max }$ and to assess if this is a stable region. For the few cases that this is possible, the continuum homology does appear as the first stable region. Moreover, rather than use random antichains, we use only minimal antichains. Again, to reduce computation times, in certain cases we calculate not the homology over $\mathbb{Z}$, but over $\mathbb{Z}_{2}$. Test examples indicate that there are no compromises because of this, since torsion appears to always be absent from these specific examples.

A test for manifoldlikeness would therefore proceed as follows: For a finite cardinality causally convex subcauset $C_{0} \subset C$ (for example, an Alexandrov interval in $C$ ), construct a random inextendible antichain $A$ in $C_{0}$, and check for the existence of stable homology regions in $T_{n}(A)$. Repeat for a statistically large enough sample. Next, check for correlations among the stable regions. If over several samplings one consistently gets a homology that is stable, then this is a good indication of manifoldlikeness. Next, if for a substantially large fraction, the homology of the first stable region is the same, then $C_{0}$ would have passed the stable homology part of our test. This, for example, suffices to demonstrate that although qualitatively the homology of causal sets generated by a class of transitive percolation dynamics appears stable and suggestive of manifoldlikeness, a statistical analysis shows that the case is significantly weaker than for the other examples studied in this paper. Specifically, the class of dynamics that we analyze is restricted by the limited 
range of parameters $(p=0.4,0.45,0.5)$ that are computationally accessible to us. Our trials show that the first stable region does not consistently give us the same homology for different inextendible antichains. Next, if the stable homology test is passed, then one needs to check for rapidly changing homology from $n=0$ to at least $n=10$ which are all uncorrelated in the different trials. The absence of such a rapidly changing region of homology for a large class of preferred antichains in the "crystalline" tower-of-crowns causal set which does have a consistent stable homology region means therefore that it is not manifoldlike.

Before proceeding to the next section we note that the above construction for homology is not unique, from a poset perspective. Indeed, posets admit a wide range of topologies [25, 26]. A more natural choice is that of chain homology for a finite sub-causal set $C^{\prime} \subset C$, which assigns to every $k$-element chain, a $k-1$-simplex. However, numerical simulations suggest that this homology, though possibly more intrinsic, does not capture the topology of the continuum. In Fig 5 we present the homology groups which arise from a sprinkling into a cylinder $S^{1} \times I$ spacetime, as described in section 3.1.2. We select an inextendible antichain at random, and give the homology groups which arise from a sequence of thickened antichains, for (a) the chain homology, and (b) the nerve. It is clear that the nerve is much more effective at capturing the topology of the continuum.

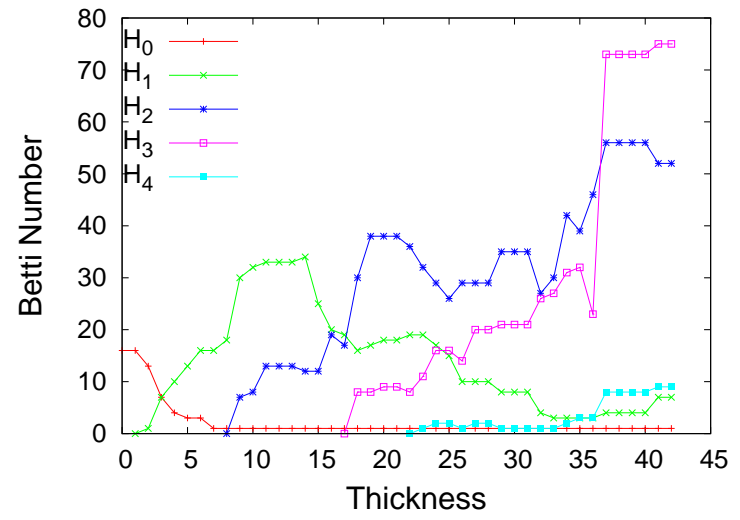

(a)

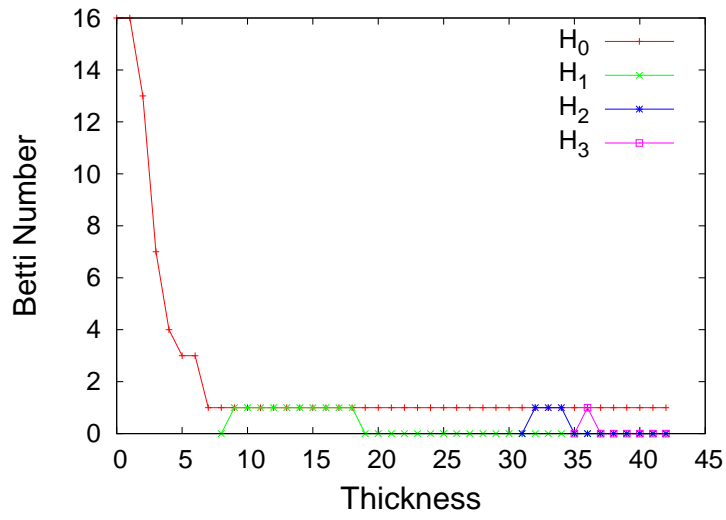

(b)

Figure 6: Plots of the Betti numbers as a function of thickness for a random antichain in an $N=258$ element sprinkling into an $S^{1} \times I$ cylinder spacetime. (a) shows the Betti numbers obtained from the chain homology of the thickened antichains, while (b) shows the Betti numbers arising from the nerve of the cover generated by the shadows cast by the maximal elements. 


\section{Results in Stable Homology}

In this section we present our main results, using the computational tools described above, for causal sets that are approximated by a class of $2 \mathrm{~d}$ and $3 \mathrm{~d}$ spacetimes. Before doing so, it is useful to first examine the choice of mesoscale $m_{s}=100$ more critically. Our subsequent analysis depends crucially on this choice, which in turn suggests that the first stable region is typically that of the continuum.

It might seem plausible that as the sprinkling density $\rho$ is increased, "spurious" regions of stable homology appear as the first stable region suggesting that $m_{s}$ needs to be modified. Would different choices of mesoscale give us different first stable homologies? The answer is yes, when we compare $m_{s}=10$ with $m_{s}=100$, since constant homology regions that are stable on scales of order the discreteness are spurious and do not reflect properties of the continuum. On the other hand, if we compare $m_{s}=100$ with an $m_{s}^{\prime} \gg 100$, then differences in the the analysis should arise only if the convexity volume (for the associated antichain) is of order $m_{s}^{\prime}$. If this is the case, then one should expect no stable regions with the choice of mesoscale $m_{s}^{\prime}$. If $m_{s}^{\prime}$ remains sufficiently smaller than the convexity volume, then the first region of stable homology will be the same as that obtained using $m_{s}$. The reason is that in our analytical understanding, there are only two scales between which the stable continuum homology is firmly wedged, namely the discreteness scale and the convexity scale6. It is therefore highly unlikely that a spurious first stable region arises before the continuum homology region. And for the same reason, if one requires $\Delta n$ to be larger than the convexity volume, then the first stable region will typically not correspond to the continuum topology. In Fig 4 these scales are shown for an antichain approximated by a $t=0$ slice in the $2 \mathrm{D}$ cylinder spacetime, with $x=0 \sim x=L$.

On the other hand a mesoscale could be determined from other expectations of the theory, like the existence of a non-locality scale [27]. In particular, if a finegrained causal set $C$ has a continuum approximation only beyond a certain coarsegraining 7 then $m_{s}^{\prime} \gg 100$ within $C$ and one should not expect continuum homology to be reproduced at smaller scales. Such causal sets will then require tests for different mesoscales. Equivalently, if $C$ is sufficiently coarse grained to some $C^{\prime} \subset C$ for which the continuum approximation should be valid, then $C^{\prime}$ should pass our test for manifoldlikeness with mesoscale $m_{s}=100$. In the causal sets we examine which are obtained by discretisations of 2 and 3 dimensional spacetimes, therefore, the lower bound for the mesoscale $m_{s}=100$ suffices.

\footnotetext{
${ }^{6}$ We can ignore the additional intermediate scale $n_{0}$ described at the end of Section 2.1, since our focus is on generic antichains.

${ }^{7} \mathrm{~A}$ causal set can be coarse grained by a process of random decimation, in which one removes some fraction of the causet elements uniformly at random.
} 
The role of the mesoscale can also be quantitatively understood by observing how $s_{R}$ varies as a function of $\rho=V_{c}^{-1}$ for causal sets obtained via sprinklings into a particular spacetime at different densities. We consider sprinkling densities of $N=1000$ to $N=8500$ in increments of 500 elements onto the unit 2 d cylinder spacetime

$$
d s^{2}=-d t^{2}+d x^{2} \quad t \in[0,1], x \in[0,1], x=0 \sim x=1 .
$$

For each of the causal sets thus obtained, we use the minimal inextendible antichain. While these antichains are not strictly coarse-grainings of each other, they are sufficiently close for our purpose. In each case, the simulations generate the continuum homology as the first stable region using $m_{s}=100$, which shows that this criterion is indeed independent of $\rho$, as long as the causal set remains manifoldlike under coarse grainings.

We moreover find a linear relation between $s_{R}$ and $N$ as shown in Fig Z(a). This relation can be understood by rewriting $s_{R}=\frac{n_{f}-n_{i}}{n_{i}}=\frac{v_{f}-v_{i}}{v_{i}}$, where the $i$ and $f$ subscripts refer to the initial and final thickening and $v_{i}=n_{i} \rho^{-1}$ and $v_{f}=n_{f} \rho^{-1}$ are the associated spacetime volumes. $v_{i}$ itself is a function of $\rho$, since as $\rho \rightarrow \infty$, $v_{i} \rightarrow 0$. If we take $v_{i}=\rho^{-1}+\mathcal{O}\left(\rho^{-2}\right)$, then to leading order, $n_{i}$ is a constant, as seen in Fig Z(b). On the other hand, $v_{f}$ being "macroscopic" is independent of $\rho$, and hence, $s_{R}=\frac{\rho v_{f}-n_{i}}{n_{i}}=a \rho+b+\mathcal{O}\left(\rho^{-1}\right)$, where $a$ and $b$ are constants. The approximation $s_{R} \approx a \rho+b$ yields a reasonable linear fit in Fig 7(a).

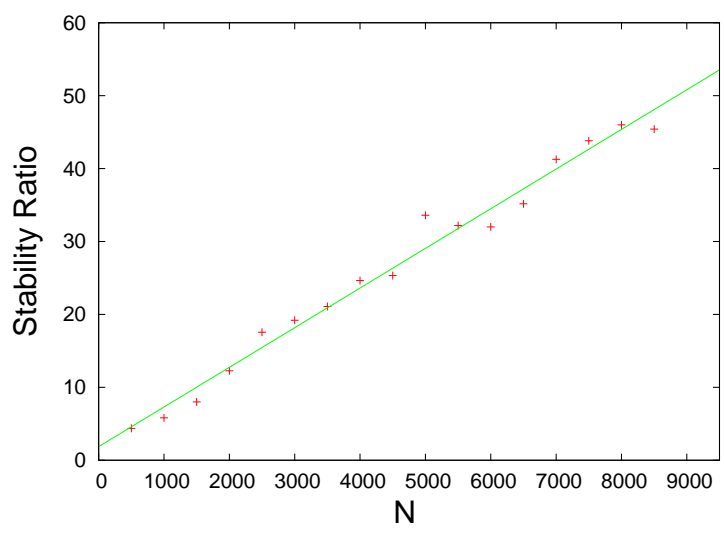

(a)

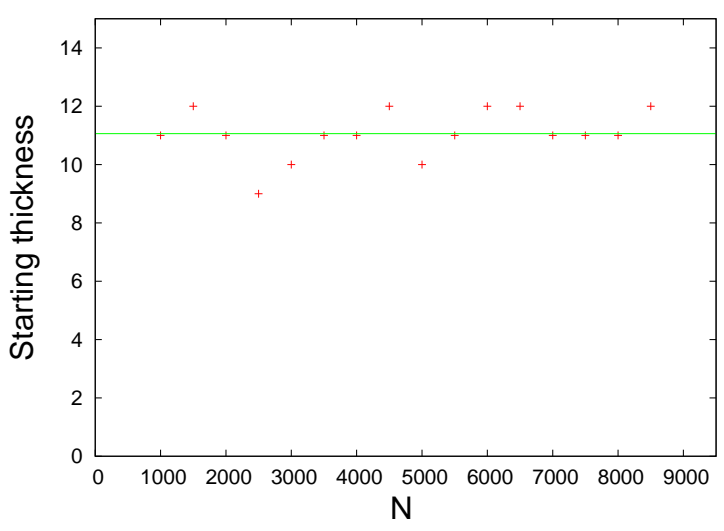

(b)

Figure 7: (a) A plot of the the stability ratio $s_{R}$ versus the inverse coarse-graining scale $N$ for the $2 \mathrm{~d}$ cylinder spacetime. (b) A plot of the starting thickness of the first stable region $n_{i}$ versus the inverse coarse-graining scale $N$. 


\section{$3.12 \mathrm{~d}$ Spacetimes}

3.1.1 Interval Spacetime: $I \times I$ with metric $d s^{2}=-d t^{2}+d x^{2}, t \in[0, T]$, $x \in\left[0, x_{1}\right]$.

This is a patch of Minkowski spacetime, and the computations involved here can be seen as a "null test" for topology. Namely, for any $(M, g)$, if the spacetime region is chosen to be small enough to be topologically (though not necessarily geometrically) trivial, then the appearance of a stable homology should correspond closely to this example. Far from being a trivial example, it characterises the topological structure of all spacetimes in the small. While the inextendible antichain obtained from a sprinkling into this spacetime region cannot be inextendible in the full space, it is nevertheless sufficient to construct the requisite localised homology. The only non-vanishing continuum homology for this spacetime is $H_{0}=\mathbb{Z}$.

We perform two different classes of computations. In the first case (a) we take $N=5000, x_{1}=1, T=1$ and thicken each randomly generated antichain up to $n_{m}=999$, or the cosmological scale $n_{\lambda}$, whichever comes first. In most of the trials, $n_{\lambda}<999$, so we indeed almost always thicken all the way to the cosmological scale. In the second case (b) we consider a much larger $N$ of 30,000 , with $x_{1}=5, T=2$. This gives us a locally dense sprinkling which makes it computationally expensive to obtain the homology groups up to the cosmological scales. However, since we are interested in establishing a correlation between the first stable region and the continuum homology, it suffices to stop the computation at some reasonably large thickness $n_{m}=499$. For each case, we repeat the calculations with 200 different random antichains on the same sprinkled causal set. Those antichains which when thickened hit the maximal elements of the causal set are thrown away in the final analysis, since we imagine physical causal sets continuing past the maximal elements of our finite simulations. 8 This reduces the overall set of useful trials. We will term a trial legitimate if the thickened antichain does not hit a maximal element before reaching $n_{m}$ or the cosmic scale. In different sets of trials for the different causal sets, we have seen the number of such legitimate trials fluctuate from 45 to 99 in 100. In order to achieve a minimum of about 100 legitimate trials we therefore find it necessary to generate 200 random antichains. We have 124 legitimate trials for (a) and 130 for (b).

In order to demonstrate the importance of the mesoscale $m_{s}=100$ we first perform the stability analysis without the additional restriction that $\Delta n \gtrsim \mathcal{O}\left(m_{s}=\right.$ 100). In (a) the continuum homology appears as the first stable region in 65 out of

\footnotetext{
${ }^{8} \mathrm{Had}$ we chosen to thicken to the past (and imagined our causal sets to continue past the minimal elements in all cases), we would have to do the same for thickenings that hit the minimal elements.
} 
the 124 legitimate trials in (b) in 75 of the 130 legitimate trials. We regard this as too poor a result. Indeed, a close examination shows that one is counting spurious stable regions which may be stable for a minimum of only two consecutive thicknesses! On the other hand, using the mesoscale of $m_{s}=100$ gives us an agreement with the continuum of $100 \%$ for (a) and 127 out of 130 for (b). In all the trials, $H_{0}$ varies rapidly from $n=0$ to $n=10$ and the behaviour is distinct for each of the trials. Also, notably, in both cases, the only non-vanishing homology group is $H_{0}$. Thus, there is only one stable region all the way up to the maximal thickness. In the set of trials (a) this is also true for the cases in which $n_{\lambda}<999$ and hence the cosmological scale is reached. We show figures from a sample trial from set (a) in Fig 8 ,

\subsubsection{Cylinder Spacetime: $S^{1} \times I$ with metric $d s^{2}=-d t^{2}+d x^{2}, t \in[0, T]$,} $x=x_{0} \sim x=x_{1}$.

This is a section of the cylinder spacetime, foliated by spatial $S^{1}$ 's. Again, as in the previous case, we consider two different sets of trials. In (a) $N=5000$ with $x_{0}=0, x_{1}=1, T=1$, and the thickening is taken up to $n_{m}=999$ or the cosmological scale $n_{\lambda}$, whichever comes first, while in (b) $N=30,000$ with $x_{0}=0, x_{1}=5, T=2$ and the thickening stops well before the cosmological scale at $n_{m}=499$. For (a), after generating the first 100 trials we found 99 of these to be legitimate and hence stopped the trials, while for (b) 200 trials yielded 155 legitimate ones. Moreover, in (a) the cosmological scale is reached for all but four of the trials, and hence one obtains a fairly global characterisation of the homology groups.

Without assuming any mesoscale, the continuum homology appears as the first stable region in only 47 of the 99 legitimate trials for (a) and 89 of the 155 legitimate trials for (b). Assuming an $m_{s}=100$ gives us a vastly improved result of 98 out of 99 for (a) and 154 out of 155 for (b). In both sets, for $n=0$ to $n=10$, the first homology group rapidly varies, and this variation is distinct in all the trials. In case (a) for all the 99 trials one sees that several higher homology groups, i.e. $H_{i}, i>2$, can become non-trivial once the first stable region is passed, but these differ from one trial to the next. In case (b) on the other hand, for all the 155 trials, only $H_{0}$ and $H_{1}$ are non-trivial. This can be attributed to the fact that the thickening stops before the end of the first stable region. Figures 9 and 10 show the homology groups as a function of thickness for one of the trials.

\subsubsection{Expanding FRW Spacetime: $S^{1} \times I$ with metric $d s^{2}=t^{2}\left(-d t^{2}+d x^{2}\right)$,} $t \in\left[T_{1}, T_{2}\right], x=0 \sim x=1$.

Although the topology is that of the cylinder spacetime, it is an important example of a spacetime with curvature. We again perform 100 computations for $N=15,000$, 


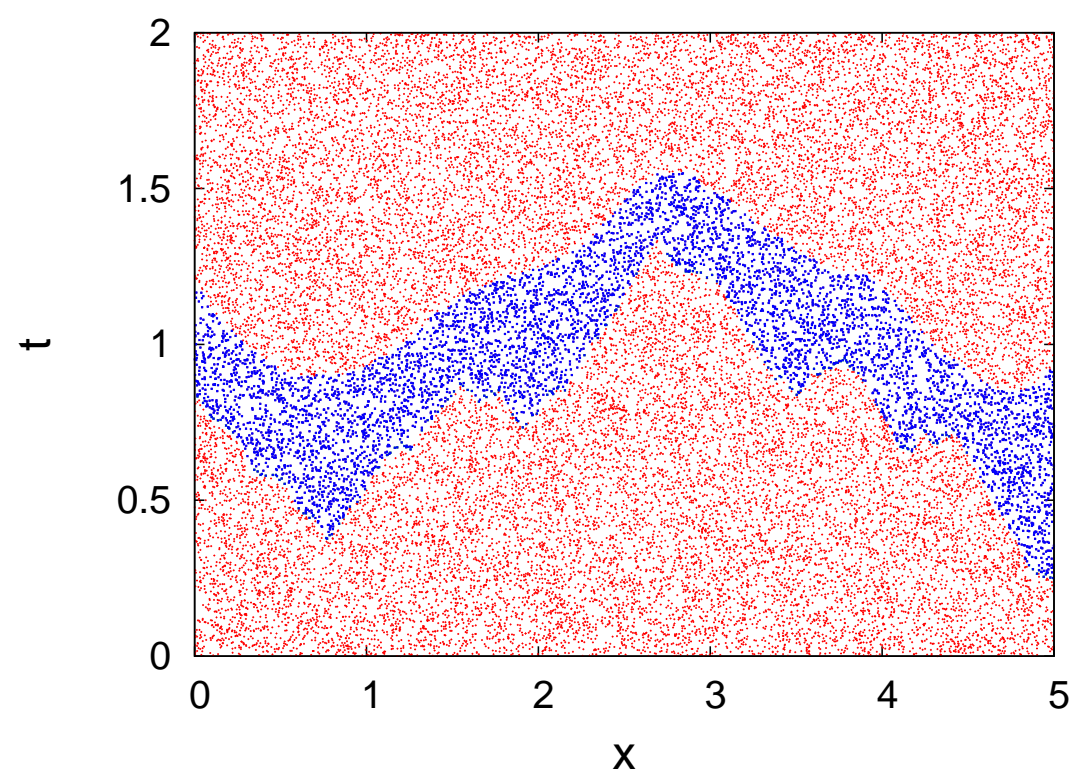

(a)

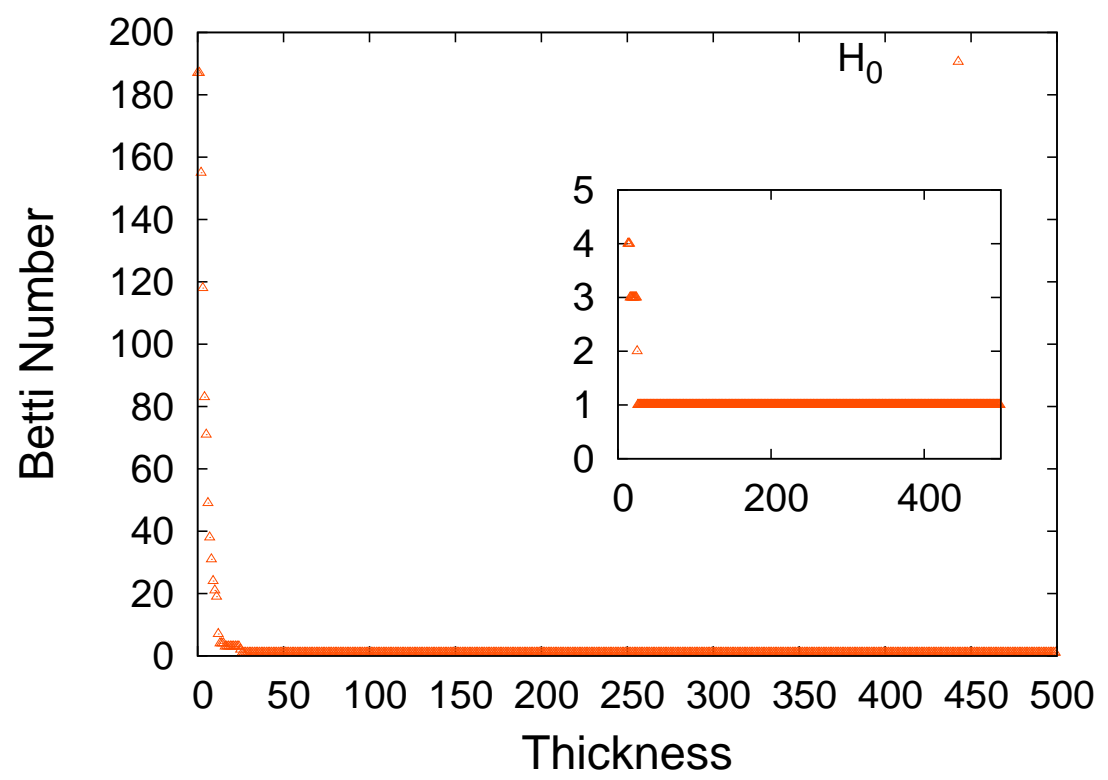

(b)

Figure 8: (a) An $N=30,000$ element causal set sprinkled into a "squat" 2d interval spacetime, with $x \in[0,5], t \in[0,2]$. A randomly chosen antichain is thickened up to $n=500$. The thickening stops well below the cosmological scale. (b) A plot of the betti numbers versus thickness. The continuum homology $H_{0}=\mathbb{Z}$ appears as the first (and only) stable region. All other homology groups vanish. 


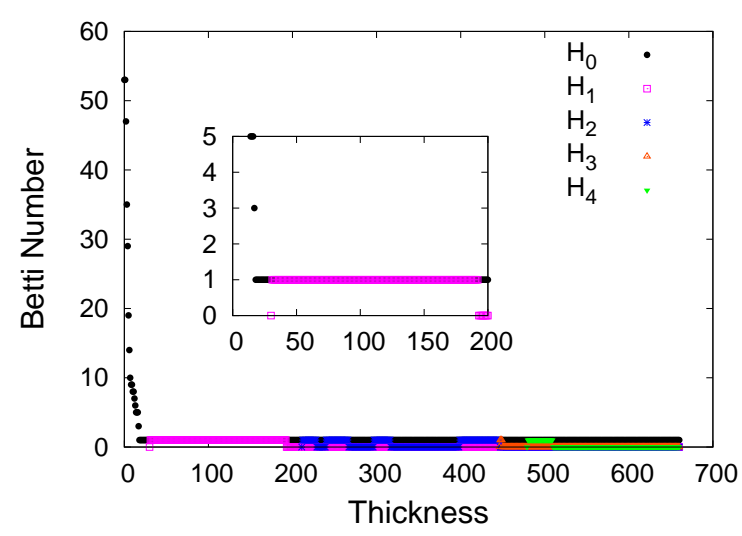

(a)

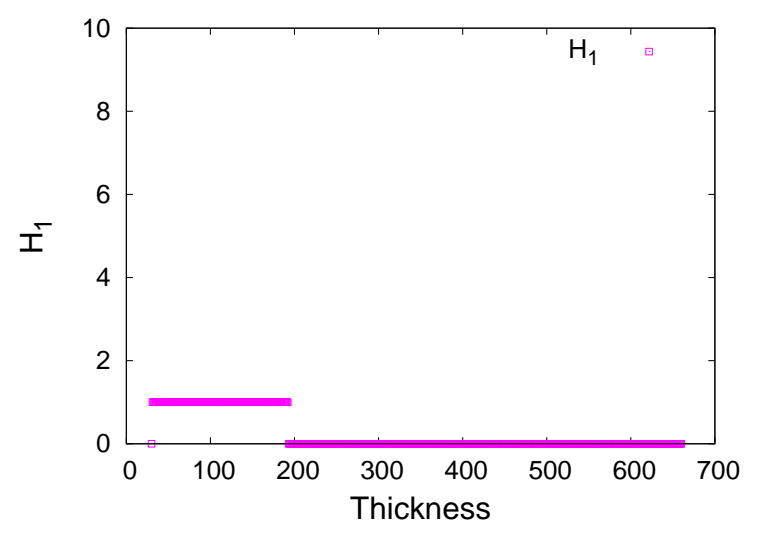

(c)

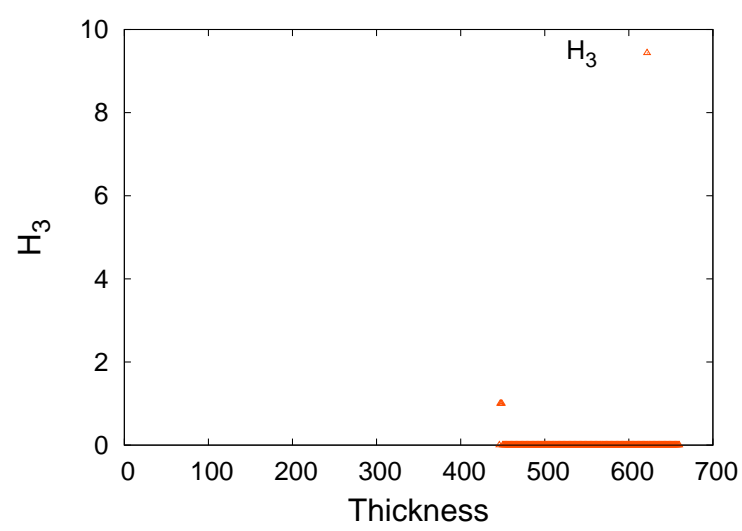

(e)

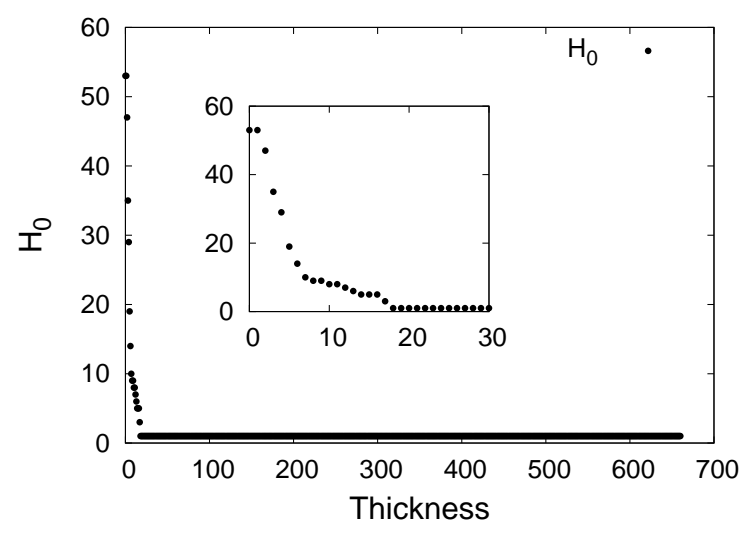

(b)

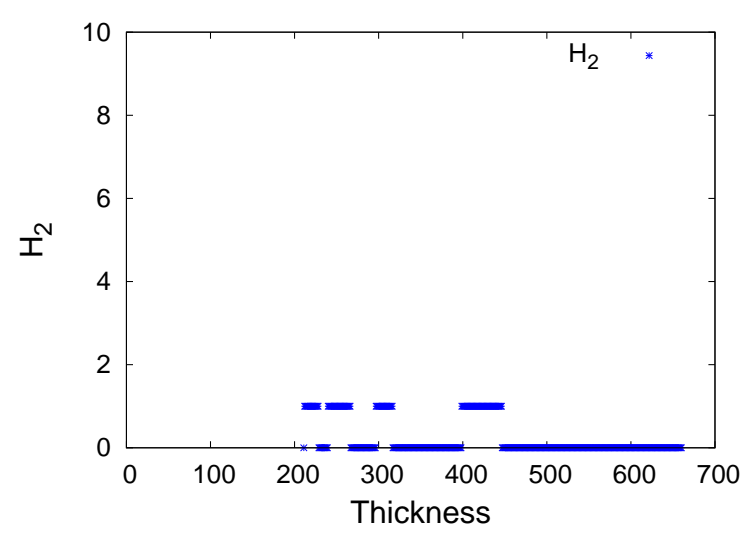

(d)

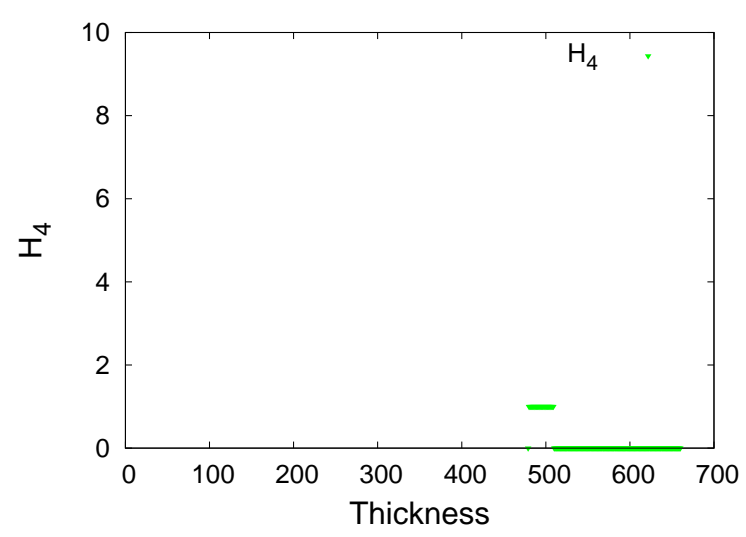

(f)

Figure 9: Homology from a random antichain in an $N=5000$ element causal set sprinkled into a (unit) $2 \mathrm{~d}$ cylinder spacetime. In (a) all the Betti numbers are plotted together as functions of thickness. (b)-(f) resolve this graph. 


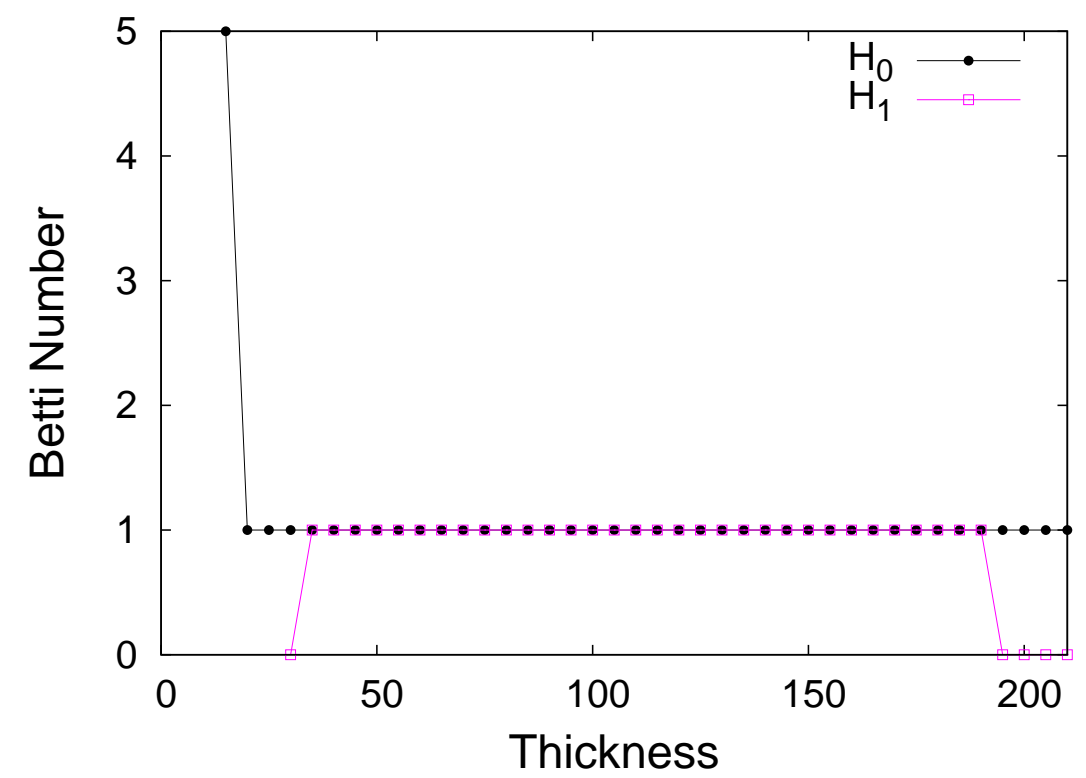

$(g)$

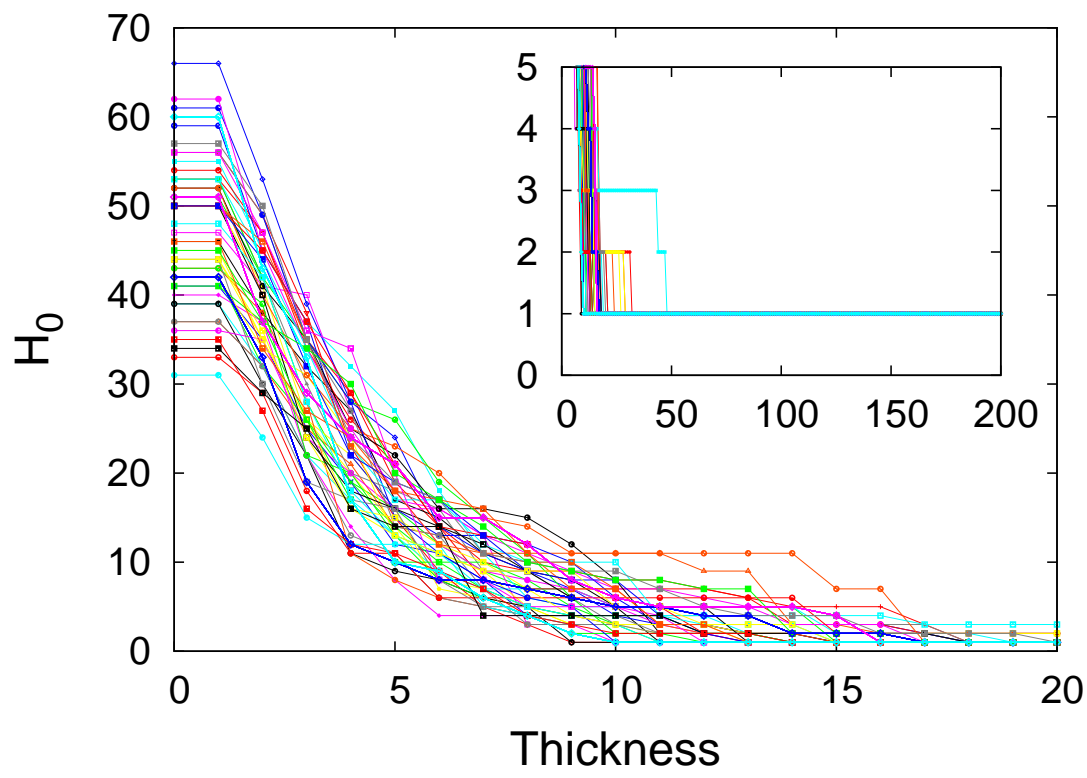

(h)

Figure 10: (g) A closer look at the stable homology region of Fig 9 from $n=12$ to $n=133$, with $H_{0}=\mathbb{Z}$ and $H_{1}=\mathbb{Z}$. (h) A plot showing the fluctuations of $H_{0}=\mathbb{Z}^{k}$ for $n \in[0,15]$ for 50 of the trials. In all cases, while $k$ decreases rapidly, the detailed behaviour is distinct for each of the trials. 
and assume a mesoscale $m_{s}=100$. The trials run up to $n_{m}=499$ or the cosmological scale $n_{\lambda}$, whichever comes first. We consider two sprinklings, (a) one for $T_{1}=0, T_{2}=$ 5 , which therefore includes the initial singularity, and (b) another for $T_{1}=4, T_{2}=6$. We find that all the trials are legitimate in both cases.

For (a) we find that the continuum homology appears as the first stable region for only 82 of the 100 trials. And for (b) this improves to 96 of the 100 trials. The lower agreement for (a) may be attributed to the existence of random antichains that lie too close to the $T_{1}=0$ singularity, thus preventing it from being manifoldlike. An example from the trials in (a) which does not reproduce the continuum homology is shown in Fig 11 as the lower antichain $A_{1}$, which exhibits only the trivial homology. Being close to the initial singularity, it has a very small cosmological scale of $n_{\lambda}=75$ which gives it no time to develop a stable spatial homology. Another example from the trials (a) which does reproduce the continuum homology is shown as the upper antichain $A_{2}$ in the same figure which, being sufficiently far away from the origin, has a larger $n_{\lambda}$ of 462 . In both sets of trials, for all trials, there is a rapidly varying region of $H_{0}$ from about $n=0$ to $n=10$, and the variation is distinct for all the trials. In case (a) 98 of the 100 trials had $n_{\lambda}<499$, so that the cosmological scale was reached. As in the cylinder spacetime, some higher homology groups (up to $H_{7}$ ) start to become non-trivial once the first stable region is passed. In case (b) only 48 of the trials reached the cosmological scale, and for 13 of these trials, one did not reach the end of the first stable region. For these, all the higher homology groups, $H_{i}$ with $i \geq 2$ are trivial.

\subsubsection{The Split Trousers Topology: $I \sqcup I \rightarrow I$}

This is the spacetime in which two disjoint intervals come together to form a single interval, so that the spatial homology $H_{0}$ transitions from $\mathbb{Z}^{2}$ to $\mathbb{Z}$ as one moves past the joint at $s_{0} \equiv\left(t_{0}, x_{0}\right)$. This is an example of a "topology changing" spacetime, i.e., where the spatial topology changes with time. Such spacetimes are not globally hyperbolic and hence the analytical results of [13] are not valid in general. Indeed, unless one admits a metric that is degenerate at $s_{0}$, such a spacetime is not even causal [28, 29, 30]. Since acausal spacetimes cannot be discretised to obtain a causal set, any topology changing spacetime of relevance to causal sets will have such isolated degeneracies. On the other hand, the very nature of causal set discretisation means that isolated points are not themselves of relevance to the causal set, which only records the coarse grained or relevant features of topology change. Hence there is a natural causal set approximation to a topology changing spacetime, which is nevertheless free of the latter's attendant pathologies.

However, the regions of the spacetime either before or after the topology changing region are globally hyperbolic, and hence the analytical results are valid here. We 


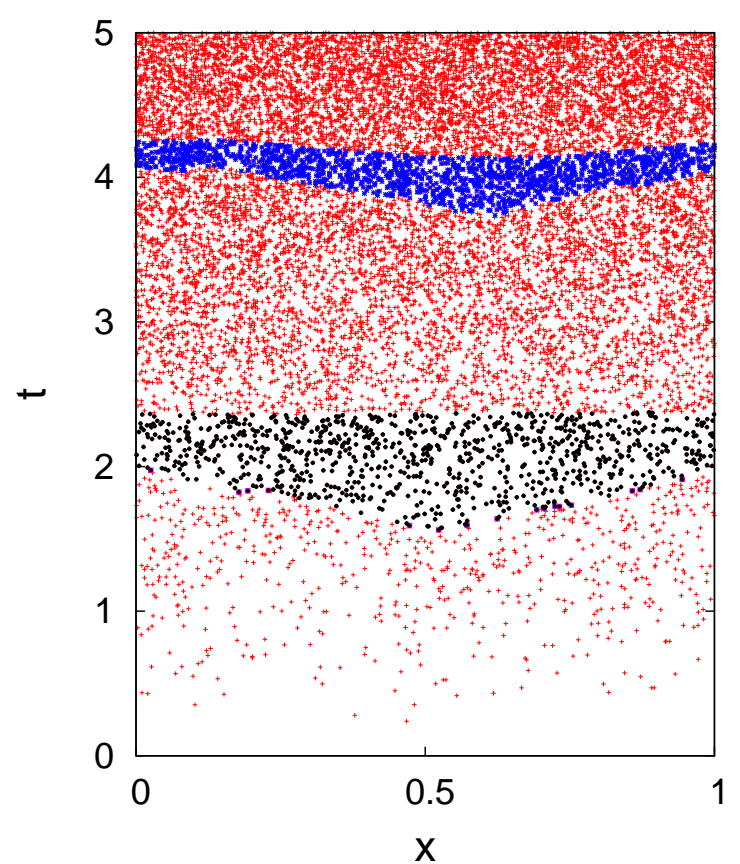

Figure 11: An $N=15,000$ element causal set obtained from sprinkling into a 2d FRW spacetime. Two different antichains $A_{1}$ and $A_{2}$ are used, and both are thickened up to their respective cosmological scales $n_{\lambda}$. Note that that for clarity, the spatial direction has been scaled up in comparison with the temporal direction. Thus, the light cones are widened. The thickened antichain $T\left(A_{1}\right)$ lies closer to the $t=0$ singularity and has a small $n_{\lambda}=75$. The continuum homology does not appear as a stable region for this trial. On the other hand the thickened antichain $T\left(A_{2}\right)$ is further from the singularity and has a much larger $n_{\lambda}=462$. It has a region of stable homology corresponding to the continuum.

consider two different sets of trials (a) and (b), both for $N=15,000$ element causal sets, obtained by sprinkling into a region (a) sufficiently before the singularity, as well as (b) sufficiently after the singularity. In both cases the singularity is at $s_{0}=(0.5,0.5)$ and one thickens up to $n_{m}=999$. For $t<0.5$, one is in the "split legs" region, with the two strips $x \in[0,0.5]$ and $x \in[0.5,1]$ and for $t>0.5$, one has the single strip $x \in[0,1]$. In set (a) of the trials $t \in[-9,1]$, so that the antichains tend to lie before the singularity, while in set (b) $t \in[0,10]$, so that the antichains tend to lie after the singularity. The number of trials in which $H_{0}=\mathbb{Z}^{2}$ appears as the first stable region is 89 out of all 89 legitimate trials for (a) and those for which $H_{0}=\mathbb{Z}$ is the first stable region is 91 out of 100 legitimate trails for (b). For 7 of the other 9 cases in (b), $H_{0}=\mathbb{Z}^{2}$ is the first stable region. Examining each such case, one notices that this is because all the antichains lie in the region before the 
transition. This difference between (a) and (b) is due to the fact that the random antichain algorithm prefers antichains that lie in the lower half of the antichain and hence the homology of the initial region with spatial topology $I \sqcup I$ is sometimes picked up. The other two cases correspond to antichains that are almost null and which lie above the transition region. Thus, they have smaller cosmological scales; for one, the cosmological scale is reached before a stable region can form, and in the other, there is an initial stable disconnected region with $H_{0}=\mathbb{Z}^{3}$ and a second stable region with $H_{0}=\mathbb{Z}$.

On the other hand, it is also interesting to focus our attention on the topology changing region and to see if our trials throw any light on it. In the simple example of the split trousers, because the only non-trivial homology group is $H_{0}$, it appears that the straddling region can actually capture the topology change. Namely, starting with an antichain that lies in $I \sqcup I$, a thickening past the singularity will connect the two $I$ 's, so that an initial region of $H_{0}=\mathbb{Z}^{2}$ is then followed by $H_{0}=\mathbb{Z}$. Since $H_{0}$ simply measures connectedness, this is clearly not sufficient; indeed, a time reversed case would not give rise to such a transition, since the connectivity on the original antichain can only increase, so that $H_{0}=\mathbb{Z}$ even past the singularity. More generally, the details of the discretisation can greatly influence the nerve for thickenings that straddle the singularity, as in the example of the stitched trousers $S^{1} \sqcup S^{1} \rightarrow S^{1}$. Pick an initial spatial hypersurface $S^{1} \sqcup S^{1}$ as in Fig [12. At two thickenings $n_{1}$ and $n_{2}$ one can pick different sets of points whose shadows give us different nerves. While the shadows from an $n_{1}$ less than the convexity volume will give the correct spatial homology $H_{0}=\mathbb{Z}^{2}$, and $H_{1}=\mathbb{Z}$ for each connected component, those from $n_{2}$ need not bear any resemblance to either of the two spatial homologies. Which of these if any would be picked out consistently in a discretisation as a second stable region is unclear.

In order to see if we can get at least a qualitative understanding of the region of topology change, we perform trials with an $N=15,000$ discretisation of the split trousers with $x \in[0,1]$ and $t \in[0,1.5]$. Again, we thicken to $n_{m}=999$ which is well below the cosmological scale. Since the topology change can no longer be ignored, the location of the initial antichain is crucial in deciphering the results. A careful examination of our trials shows that for thickened antichains which straddle the region of topology change, a first stable region with $H_{0}=\mathbb{Z}^{2}$ is followed by a second with $H_{0}=\mathbb{Z}$. If the antichain is chosen too close to the transition, or well above it, only the final topology shows up as a stable region. We get 97 legitimate trials. Of these, we find that a first stable region of $H_{0}=\mathbb{Z}^{2}$ followed by a second stable region of $H_{0}=\mathbb{Z}$, occurs in only 29 times out of the 97 trials. By itself, however, $H_{0}=\mathbb{Z}^{2}$ occurs as the first stable region in 56(including the 29 above), while $H_{0}=\mathbb{Z}$ occurs as the first stable region in 32 of them. The bias towards the former is related to our choice of picking antichains in the lower half of the bounding box, to avoid 


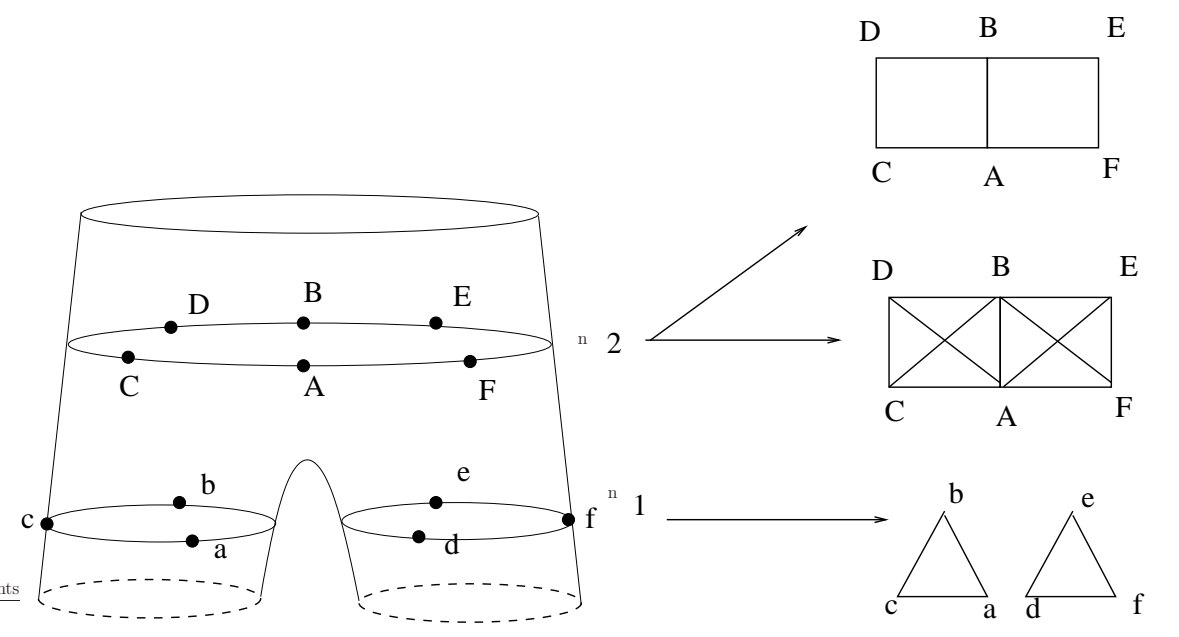

Figure 12: The stitched trousers topology $S^{1} \sqcup S^{1} \rightarrow S^{1}$. Starting from a hypersurface in the region before the topology change with topology $S^{1} \sqcup S^{1}$ (shown as two dashed circles) the correct homology $H_{0}=\mathbb{Z}^{2}, H_{1}=\mathbb{Z}$ for each connected component is obtained for thickenings $n_{1}$ that lie below the convexity volume. For $n_{2}$ larger than this volume, and past the singularity, depending on how the discretisation is done, one can get different homologies, none of which bear any relevance to either of the spatial topologies. Thus, the first nerve on the right has $H_{0}=\mathbb{Z}$ and $H_{1}=\mathbb{Z}^{2}$, while the second (which represents the boundaries of two tetrahedra joined at an edge) has $H_{0}=\mathbb{Z}, H_{1}=0$ and $H_{2}=\mathbb{Z}^{2}$.

running into maximal elements. If instead we ask if $H_{0}=\mathbb{Z}^{2}$ or $H_{0}=\mathbb{Z}$ occur as stable regions, with the condition that if only the first or second appear, then they must be the first stable region, and if they occur together they must appear one after the other, this occurs in 87 of the trials. In general, though, it would seem difficult to assess manifoldlikeness for a topology changing region, without additional restrictions on the coarse-grained locations of the random antichains used. Fig 13 shows three trials in which the thickened antichain straddles three different topological regions of the spacetime.

We should reiterate here that although the region of topology change is not globally hyperbolic, when we do restrict to the globally hyperbolic regions in the spacetime, as in case (a) and (b) above, we obtain the correct continuum homology of these regions. However, given a causal set $C$, our random antichain algorithm is not currently suited to pick out the different globally hyperbolic regions. One way to achieve this would be to construct a partially ordered set from the set of all possible inextendible antichains of $C$, such that an antichain $A$ precedes another $B$ iff no element of $B$ precedes an element of $A$. A chain in this poset would correspond to a "foliation" of the causal set by inextendible antichains. Such a foliation would 


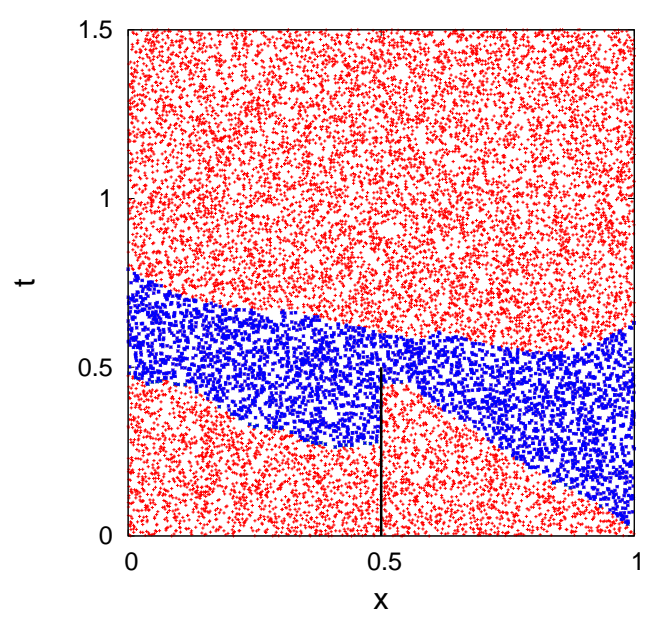

(a)

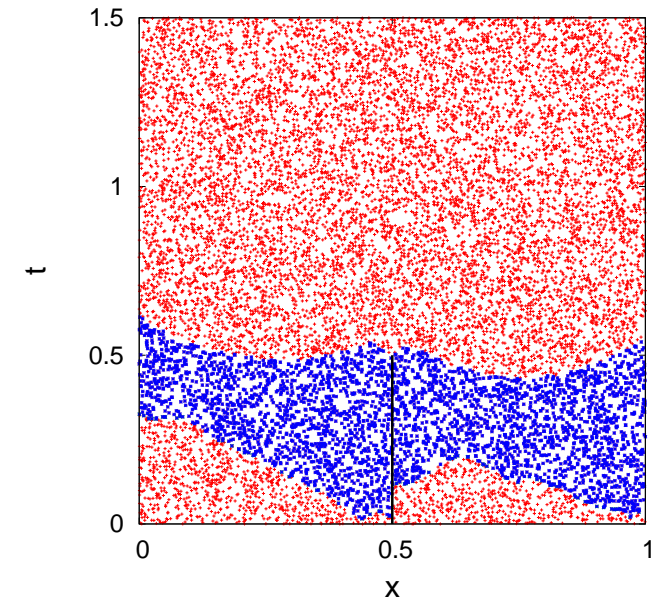

(b)

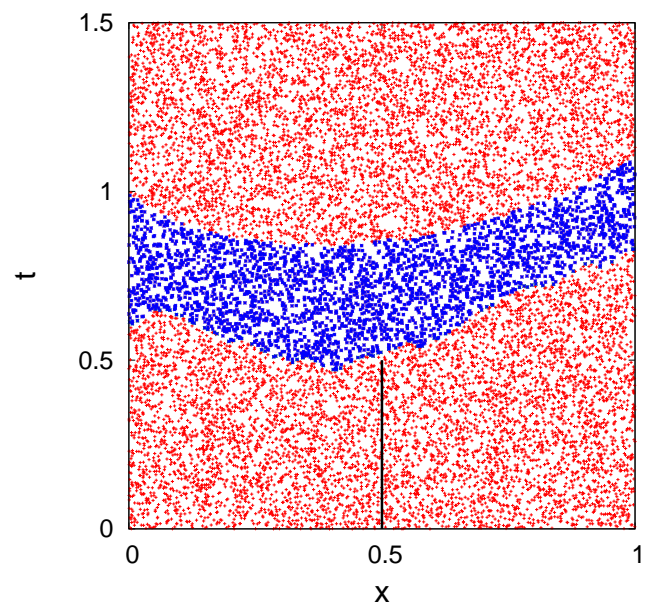

(c)

Figure 13: These are examples from the sprinklings into the trousers spacetime with $N=15,000, x \in[0,1]$, and $t \in[0,1.5]$ (a) The thickened antichain straddles the region of topology change, so the first stable region has $H_{0}=\mathbb{Z}^{2}$ followed by a stable region $H_{0}=\mathbb{Z}$. (b) This thickened antichain lies below the region of topology change, and so the first stable homology region with $H_{0}=\mathbb{Z}^{2}$ is not followed by any other stable region. (c) In this case, the thickened antichain lies above the topology changing region, so the only stable homology is $H_{0}=\mathbb{Z}$. 
not be difficult to generate, by a variety of methods. Thickening the antichains in a foliation, one could find the stable homology as a function of the foliation parameter, and hence isolate the various globally hyperbolic regions in $C$ if they exist. This procedure is computationally intensive, but can be carried out in principle.

\section{$3.23 d$ Spacetimes}

Although it is relatively easy to generate the nerve in higher dimensions, the homology algorithm CHomP slows down considerably because of the large number of high dimensional simplices that are generated. Thus, a statistical analysis along the lines carried out for the $2 \mathrm{~d}$ examples is not possible, and we will use the homology calculations to reinforce qualitatively what we have already observed in the $2 \mathrm{~d}$ examples. On the other hand, the $3 \mathrm{~d}$ computations also allow us to consider a compactified direction whose size can be varied with respect to the discreteness scale, thus studying the effects of coarse graining on the region of stable homology. It helps speed up our computations to use the preferred minimal antichain, which is what we will do in all of the trials. For some of the examples we also calculate homologies over $\mathbb{Z}_{2}$ instead of $\mathbb{Z}$, which again cuts down the run times considerably. This would suggest a reduction of information, but several tests comparing the two do not find any differences. Figures 14 show this for a specific example.

Before we proceed with the examples it is useful to explain the choice of simulations that we exhibit here. Although several tens of $3 \mathrm{~d}$ trials were started, many had to be terminated, because they could not be run to completion within reasonable time. Estimates of run times using different parameters were made and a process was deemed too slow (using the RRI-AMD cluster of machines), if it took more than a month to compute up to $n=25$. For low sprinkling densities, of course, the run time is also shorter. However, the only stable homology is the trivial one for these low density trials, so the continuum does not manifest itself at all for the non-trivial topologies. A handful of trials therefore remained which provided results of value. The ones we have picked from these show something more definitive than the others. It is important to stress that none of the others contradict the basic hypothesis, but we do not discuss them here because they either are not as complete or the sprinkling density is too small.

We examined the following three spacetime topologies:

(a) The 3d Minkowski interval spacetime with topology $I \times I \times I: d s^{2}=-d t^{2}+$ $d x^{2}+d y^{2}, t \in[0,1], x \in[0,1], y \in[0,1]$. We calculate the homology over $\mathbb{Z}$ as in the $2 \mathrm{~d}$ case, because of the relative simplicity of the homology. We show in Figures 15 and 16 examples with low sprinkling densities, $N=1024$ and $N=2048$. In both cases we see that the first stable region is indeed the continuum one, with the choice 


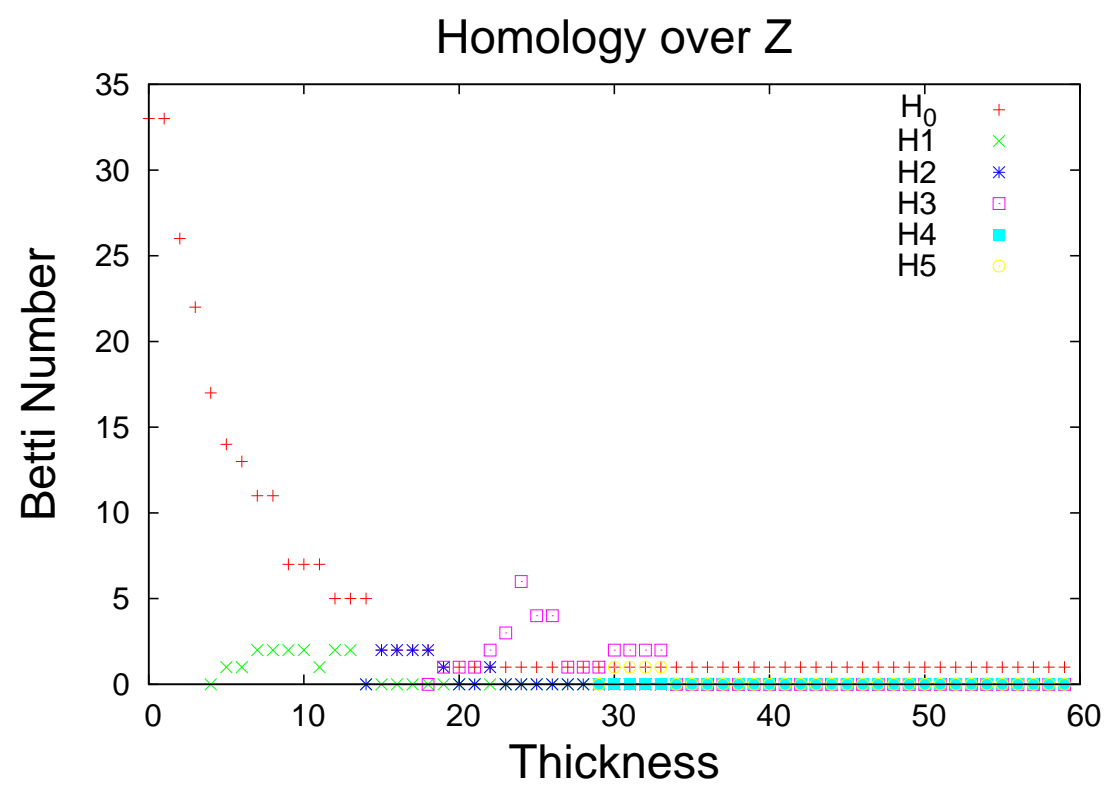

(a)

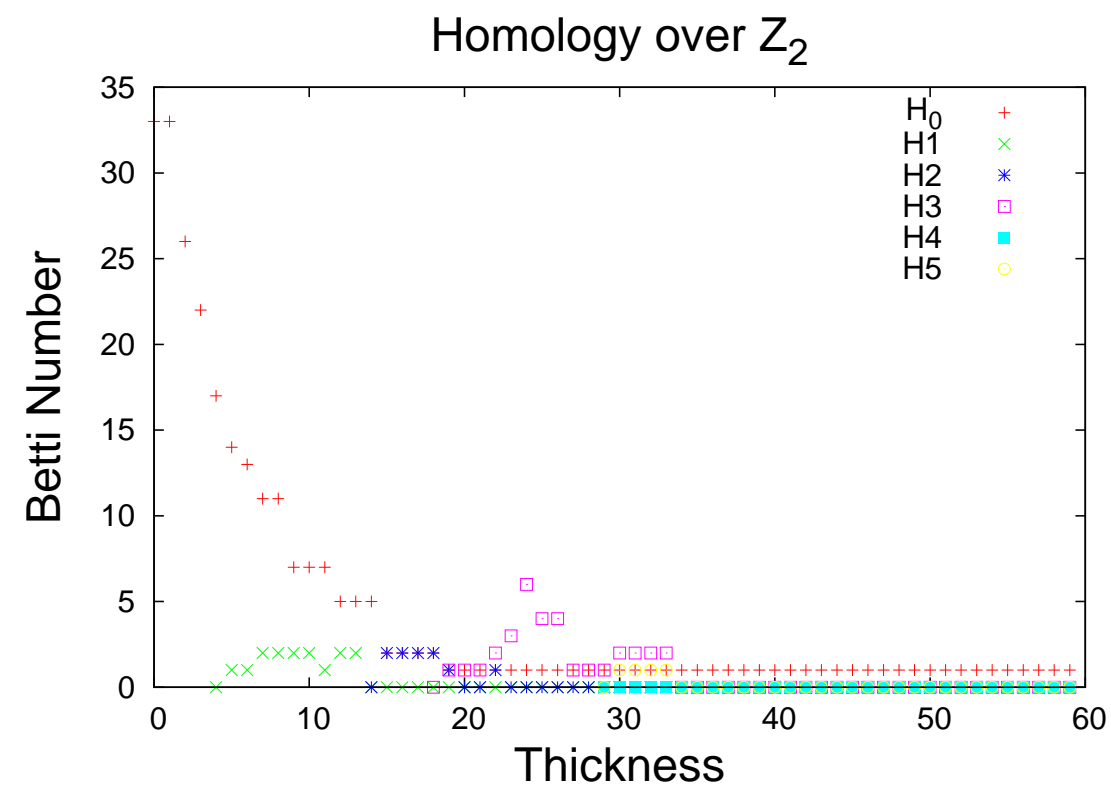

(b)

Figure 14: Comparison of homology calculations for a causal set approximated by a $T^{2} \times I$ spacetime, with $N=1000$. (a) uses homology on $\mathbb{Z}$ and (b) uses the homology on $\mathbb{Z}_{2}$. The former exhibits no torsion, so their Betti numbers suffice and exhibit no difference. 
of mesoscale $m_{s}=100$ as in the $2 \mathrm{~d}$ case.

(b) The $2 \mathrm{~d}$ Minkowski interval spacetime with an $S^{1}$ compactified direction, with $t \in[0,1], x_{1} \in[0,1]$ and $x_{2} \in[0,1], x_{2}=0 \sim x_{2}=1$. The trials were carried out for $N=1024, N=2048$ and $N=4096$, and the computation proceeded well beyond the stable region. The homology over $\mathbb{Z}$ was computed. In all three cases, the first stable region is that of the continuum, i.e., $H_{0}=\mathbb{Z}, H_{1}=\mathbb{Z}$, again with $m_{s}=100$. We show one of these examples in Fig 17 where $N=2048$.

(c) The spacetimes with topology $T^{2} \times I: d s^{2}=-d t^{2}+d x^{2}+d y^{2}, t \in[0,1], x \in[0, a]$ with $x=0 \sim x=a, y \in[0, b]$ with $y=0 \sim y=b$. Trials were carried out for (i) $a=b=1$ (Fig 18) and (ii) $a=0.25, b=1$ (Fig 19). In all cases, the homology over $\mathbb{Z}_{2}$ was computed. In (i) the simulations gave the best results for an $N=4096$ causal set. In this case, the $3 \mathrm{~d}$ homology begins to appear as a constant homology region, but the simulations could not be run beyond this stage. For lower densities $N=1024$ and $N=2048$, these continuum homology regions appear fleetingly, and hence are not stable. For (ii), trials were carried out for $N=1024$ and $N=2048$ and for both the $2 \mathrm{~d}$ cylinder spacetime homology $H_{0}=\mathbb{Z}_{2}$ and $H_{1}=\mathbb{Z}_{2}$ appears as the first stable region, again with $m_{s}=100$. The $3 \mathrm{~d}$ region does not appear even fleetingly in these cases. The low sprinkling densities mean that the small compactified direction $S^{1}$ does not appear as a continuum feature in the causal set.

\section{Testing for Manifoldlikeness}

We now consider two classes of causal sets neither of which is obtained via a causal set discretisation of a spacetime. The first class contains causal sets that are obtained from a particular classical growth dynamics called "transitive percolation". The second is a class of regular or crystalline causal sets, the "tower of crowns". Since they do not arise from a spacetime discretisation, they are ideal candidates to put through our test for manifoldlikeness.

\subsection{Transitive Percolation}

As a precursor to understanding the quantum dynamics of causal sets, a class of classical sequential growth dynamics for causal sets was constructed in [5]. One starts with a single element and adds new elements one at a time such that at stage $n+1$ the new element cannot be added to the past of any element in the $n$-element causal set obtained at the previous stage. The process is Markovian and is required to satisfy the physical criteria of label invariance and a "Bell-causality" 


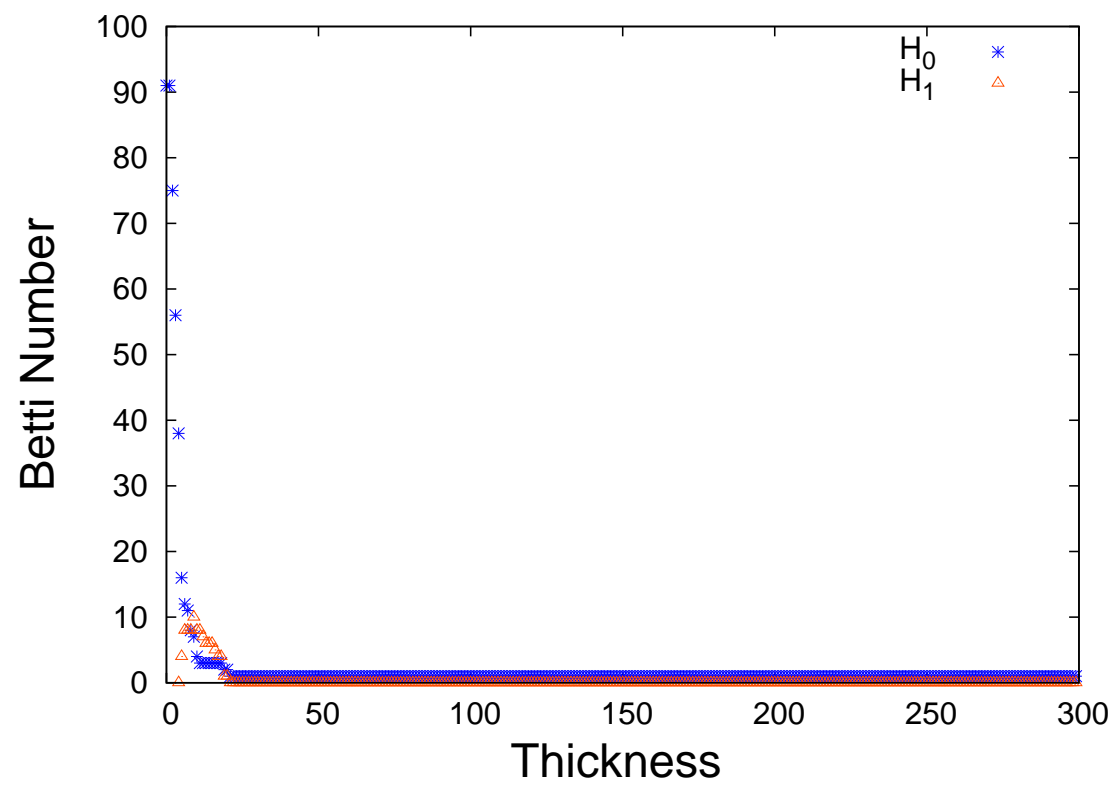

(a)

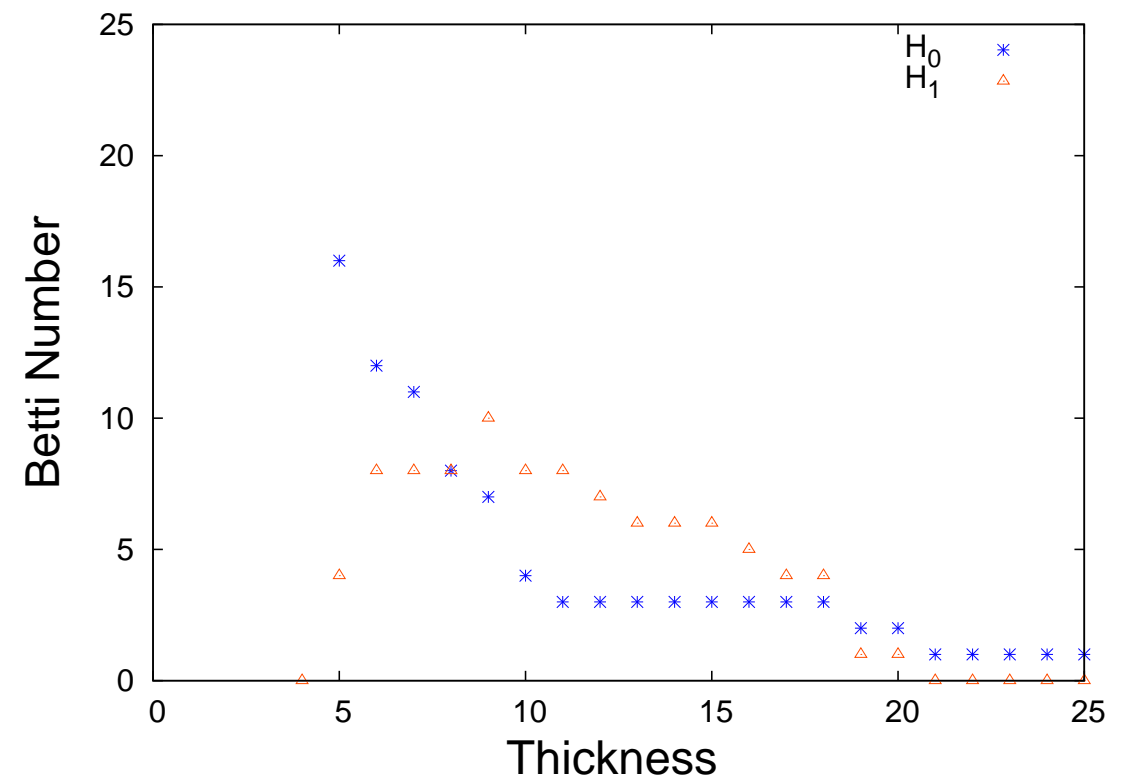

(b)

Figure 15: (a) The stable homology for an $N=1024$ element causal set obtained from a sprinkling into a $3 \mathrm{~d}$ interval spacetime. The first stable region is that of the continuum. (b) For small $n$ the homology is rapidly varying. 


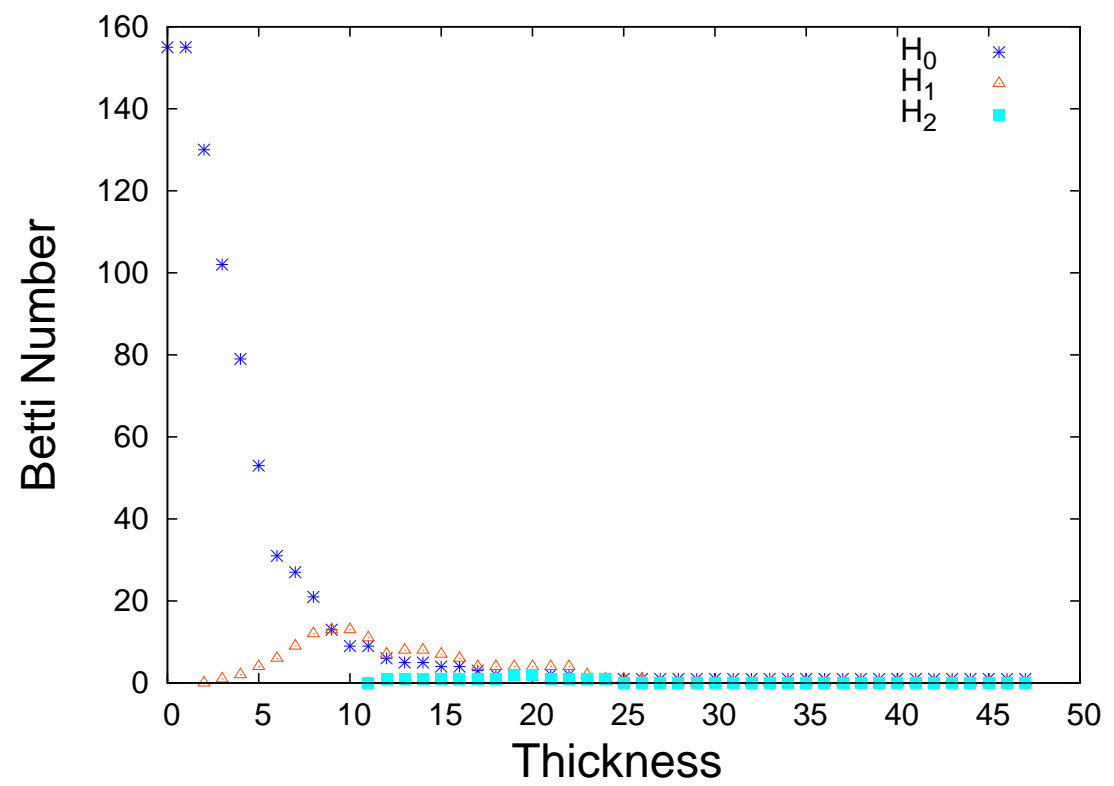

(a)

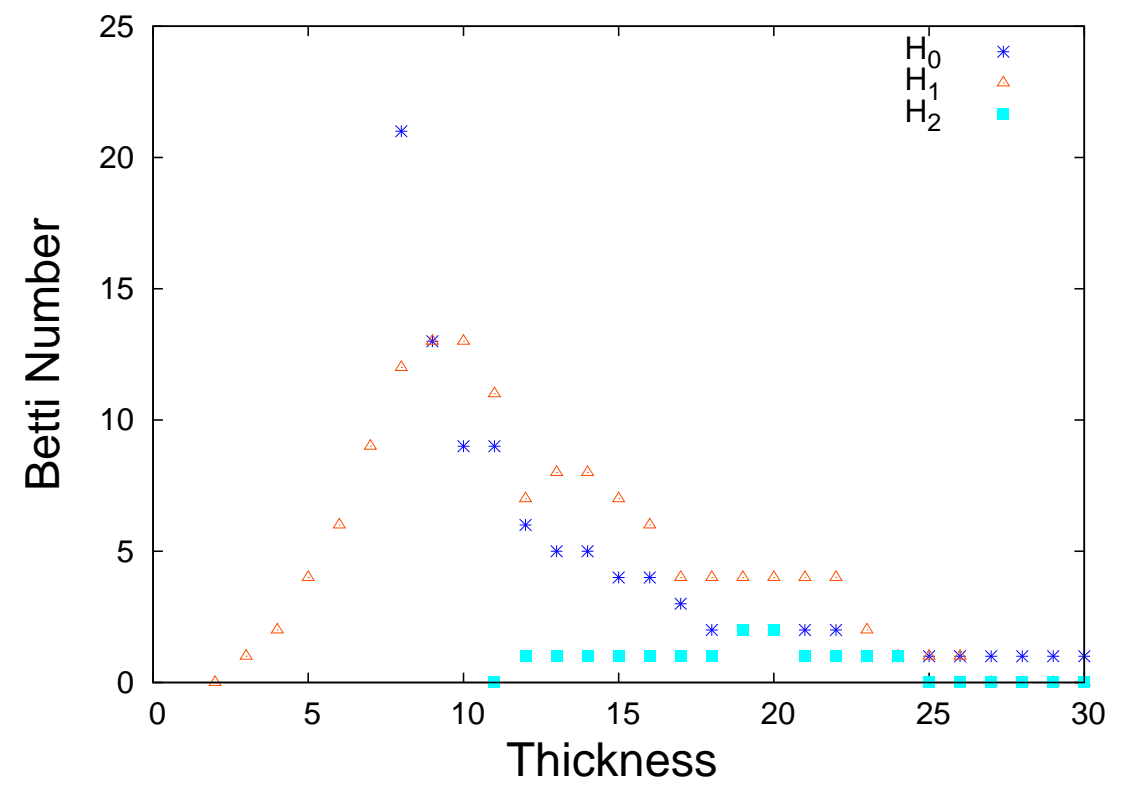

(b)

Figure 16: (a) The stable homology for an $N=2048$ element causal set obtained from a sprinkling into a $3 \mathrm{~d}$ interval spacetime. The continuum homology begins to appear as a constant homology region. (b) For small $n$ the homology is rapidly varying. $H_{2}$ is also non-zero in this region, unlike the $N=1024$ case. 


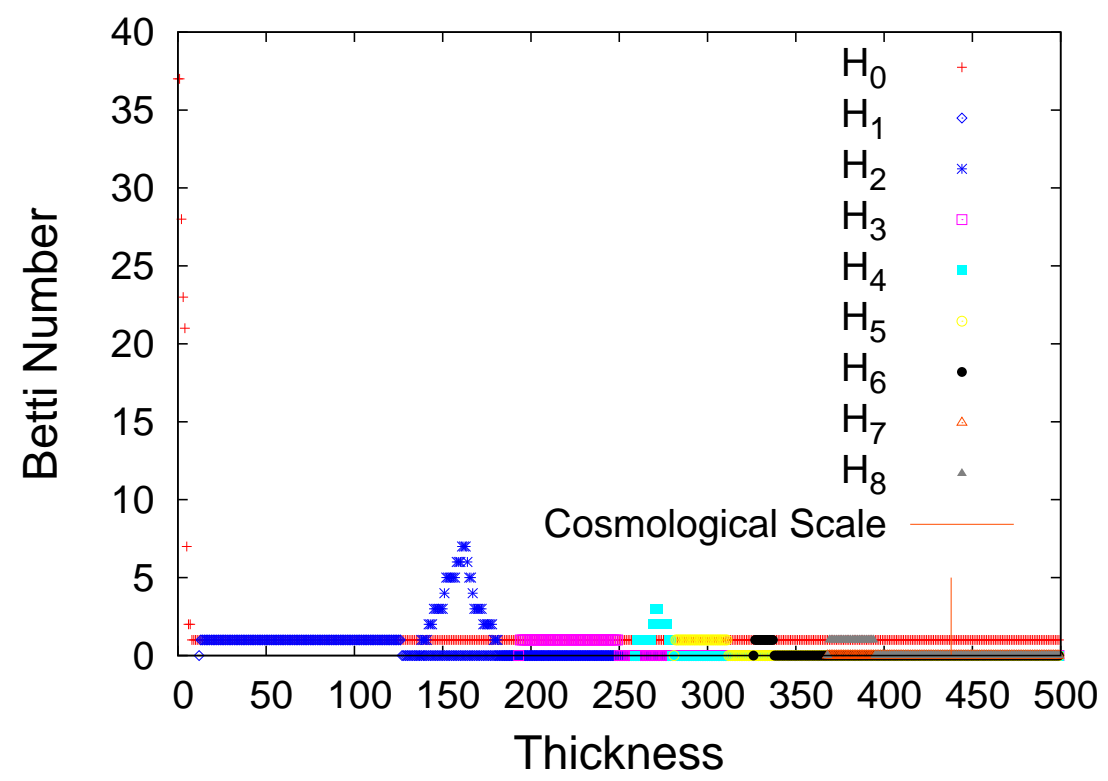

(a)

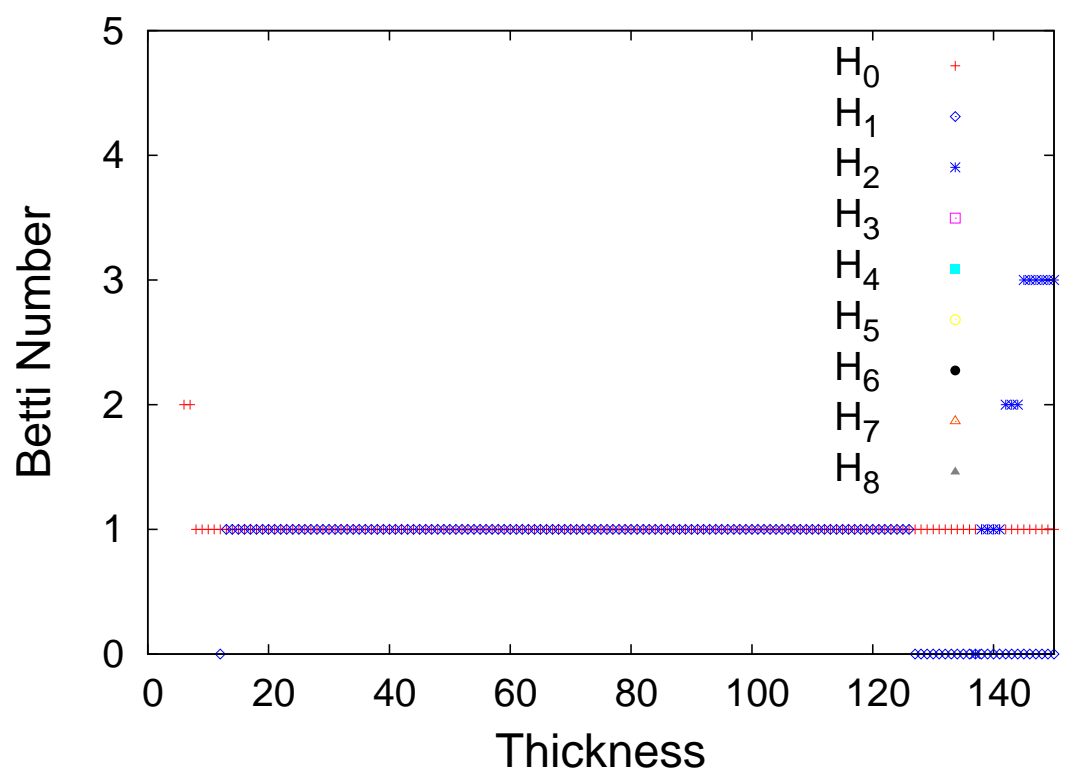

(b)

Figure 17: (a) The homology for a minimal antichain for an $N=2048$ element causal set obtained from the $S^{1} \times I \times I$ spacetime. (b) Close up of the first stable homology region which agrees with that of the continuum. 


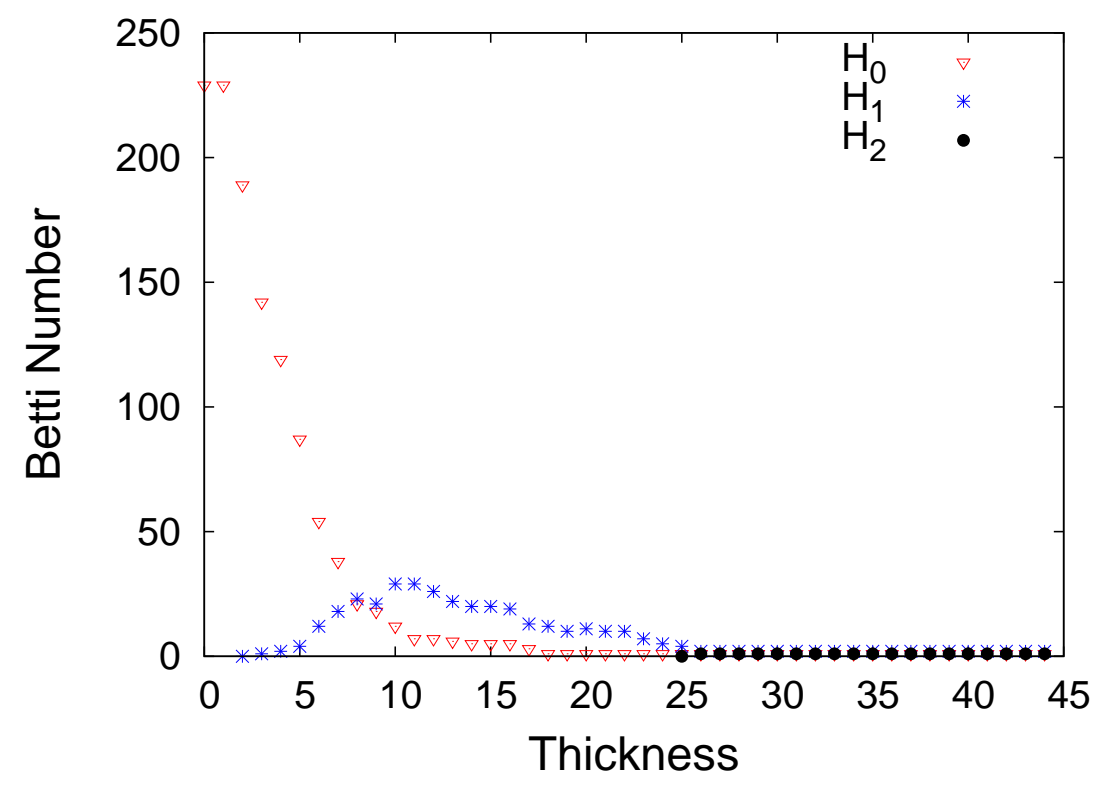

(a)

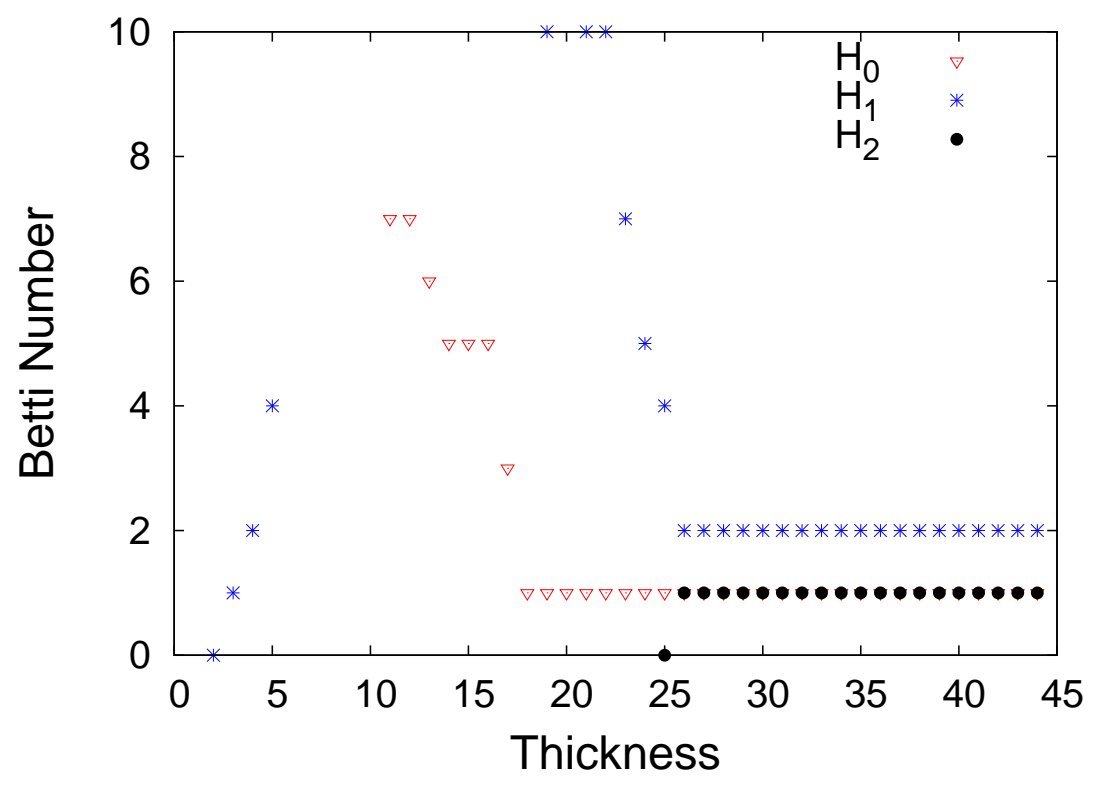

(b)

Figure 18: (a) An $N=4096$ causal set obtained from sprinkling into a $T^{2} \times I$ spacetime, with $a / b=1$. From $n=26$ to $n=44$ the homology is that of $T^{2}$ with $H_{0}=\mathbb{Z}, H_{1}=\mathbb{Z}^{2}$ and $H_{2}=\mathbb{Z}$. The computation was stopped before the end of this region could be found. (b) A closer look. 


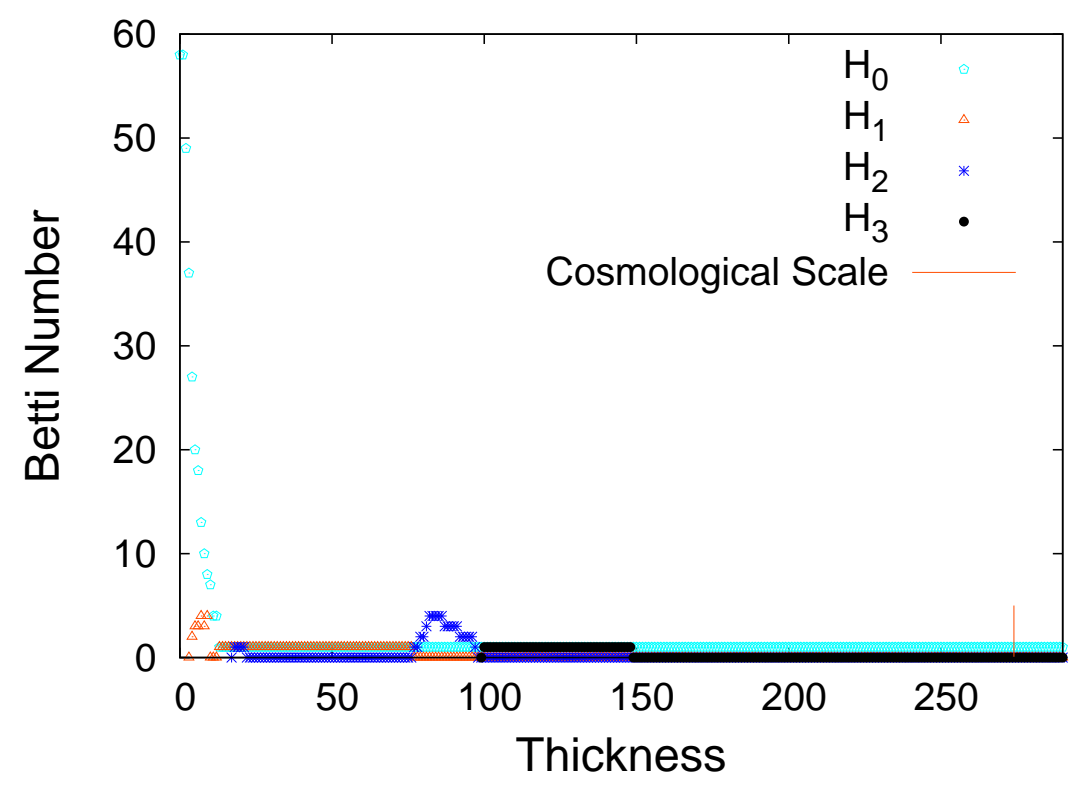

(a)

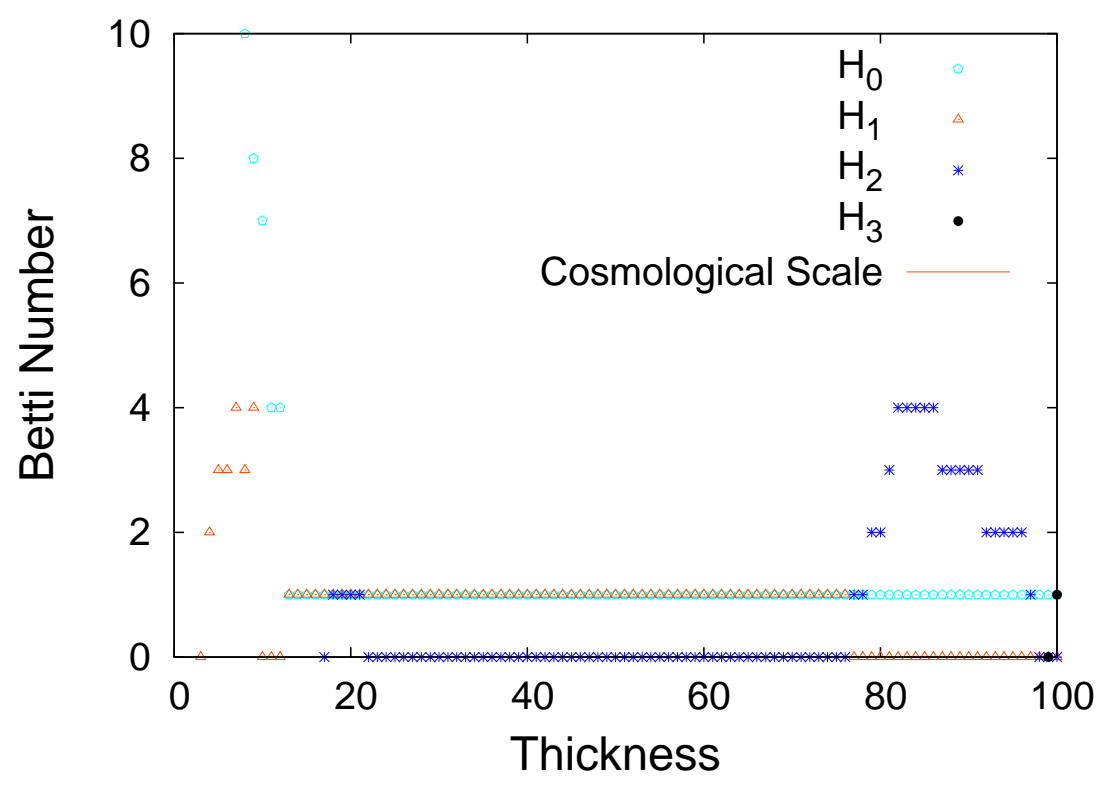

(b)

Figure 19: An $N=1024$ element causal set sprinkled into the $T^{2} \times I$ spacetime with aspect ratio $a / b=0.25$. The $2 \mathrm{~d}$ homology groups $H_{0}=\mathbb{Z}, H_{1}=\mathbb{Z}$ appears as the first stable region. The $3 \mathrm{~d}$ homology is not captured even fleetingly. 
condition [5]. These criteria restrict the dynamics to a class of "general percolation" models studied in detail in [5, 18, 31], of which transitive percolation is of particular interest. In [18, 19, 20] it was shown that in a cosmological context, the causal set can undergo a sequence of bounces, with each era between bounces determined by a different generalised percolation dynamics. At late times, it was shown that the parameters "flow" to that of transitive percolation. Hence it is of interest to see if a typical causal set obtained via transitive percolation has any manifoldlike characteristics.

The transitive percolation dynamics is determined by a single parameter $0 \leq$ $p \leq 1$ which gives the probability for a new element at stage $n$ to be linked to an element of the $n$-element causal set obtained at stage $n-1$. Thus, starting from a single element, the probability to get the 2-element chain is $p$ and the 2-element antichain is $1-p$, that of a 3 -chain is $p^{2}$, that of a 3 -antichain $(1-p)^{3}$, etc.

We run our trials for the following three choices of $p=0.05,0.045,0.04$ for $N=4000$ element causal sets. The reasons for these choices are that (i) for larger $p$, the cosmological scale appears very quickly, and our trials suggest that there are typically no stable regions at all before the cosmological scale 9 and (ii) for smaller $p$, the run-times become too large to perform a suitable statistical sampling. However, it is known that the number of elements in a largest inextendible antichain, or "width", of a causal set generated by transitive percolation $\sim 1 / p$. Thus, the transitive percolated causal sets we consider are quite narrow, with a width of no more than $\sim 1 / .04=25$. Therefore in this sense we are only able to access relatively small transitive percolated causal sets computationally, and so may not be surprised that they have trouble producing regions of stable homology beyond the mesoscale, but before the cosmic scale sets in.

For each case, we perform 100 trials. For $p=0.05$, all the trials are legitimate, although 49 of these have no stable regions. A case by case analysis reveals that this is because the cosmological scale is very small for each of these trials. We find that 44 of the trials have $H_{0}=\mathbb{Z}$ as the first stable region, 7 with $H_{0}=\mathbb{Z}^{2}$, the other homology groups all being trivial. In none of these cases is there more than one region of stable homology before the cosmological scale.

For $p=0.045$, again all 100 trials are legitimate. We find that 62 of the trials have $H_{0}=\mathbb{Z}$ as the first stable region, 16 with $H_{0}=\mathbb{Z}^{2}$, the other homology groups all being trivial. In addition, 2 of these have first stable region $H_{0}=\mathbb{Z}^{2}$ and second stable region $H_{0}=\mathbb{Z}$, while 22 have no stable regions.

For $p=0.04$, we find that now only 53 of the 100 legitimate trials have $H_{0}=\mathbb{Z}$ as the first stable region, 30 have $H_{0}=\mathbb{Z}^{2}$, and only 1 of these 30 has the first stable region with $H_{0}=\mathbb{Z}^{2}$ followed by a second stable region with $H_{0}=\mathbb{Z}$. There

\footnotetext{
${ }^{9}$ For $p=0.1$, for example, out of 100 trials, only 6 had stable regions.
} 
is only one other case with stable first stable homology $H_{0}=\mathbb{Z}^{3}$ followed by a second stable region of $H_{0}=\mathbb{Z}^{2}$. There are 16 trials with no stable regions. Fig 20 shows an arbitrary sample of the homology groups which arise from an inextendible antichain from transitive percolation at $p=0.04$. The $H_{0}=\mathbb{Z}^{2}$ homology occurs as a stable region for this antichain. Some of the higher homology groups, up to $H_{4}$, become non-trivial before the first stable region begins, thus contributing to the "discreteness noise". This is a feature we do not see in the causal sets obtained from $2 \mathrm{~d}$ discretisations.

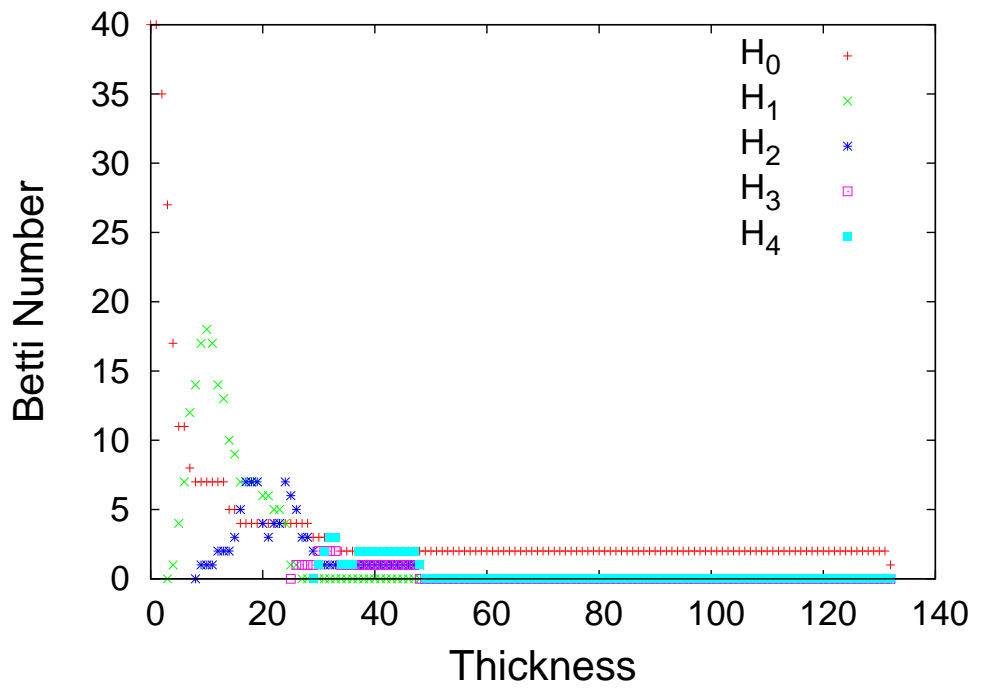

Figure 20: A sample of the homology groups for $p=0.04$ transitive percolation with $N=4000$. The thickness parameter runs all the way to the cosmological scale.

Thus, it appears that for these values of $p$, the causal sets do not pass our test for manifoldlikeness with sufficient confidence, although the appearance of stable regions, along with an initial period of instability seem suggestive. It is tempting to conclude that these results are compatible with the split trousers topology in the region of topology change. There are definitely similarities, especially with the $p=0.45$ case. If the existence of a second stable region before the cosmological scale is an indicator of topology change on the other hand, there are only 6 instances of a $H_{0}=\mathbb{Z}^{2}$ to $H_{0}=\mathbb{Z}$ transition. If the comparison to the inverted trousers had to be made, then as argued earlier, there can be no antichains for which such a transition can be made. However, it does seem plausible that one can "tailor" a trousers spacetime to suit the outcome of this trial. On the other hand, it is important to be cautious in this comparison. Neither the trousers nor these cases of transitive percolation satisfy our test of manifoldlikeness. Further work on stable homology in topology changing spacetimes is required for us to conclude that this similarity is more than incidental. 


\subsection{Tower of Crowns}

It is useful to consider as an example a regular causal set, the tower of crowns, which embeds into the $2 \mathrm{~d}$ cylinder spacetime, though not faithfully. Its regularity means that it has regions of stable homology. In this case, this homology is that of $S^{1}$, and hence it seems to pass one of the tests for manifoldlikeness. However, its regularity also means that for small thickenings, the fluctuations are not sufficiently uncorrelated. The simplicity of the causal set allows us to examine it analytically.

A crown is a 2 layer causal set, with $m>2$ elements in each layer. It has a natural labelling in terms of which the order relations can be expressed. Namely, if $\left\{e_{1}(0), e_{2}(0) \ldots e_{m}(0)\right\}$ are the elements in the bottom layer and $\left\{e_{1}(1), e_{2}(1) \ldots e_{m}(1)\right\}$ in the top layer, then $e_{i}(0) \prec e_{i}(1)$ and $e_{i}(0) \prec e_{i+1}(1)$ with the labels $i \equiv i \bmod m$ (Fig 4.2(a)). Stacking an infinite number of m-crowns one on top of the other gives us the tower of crowns (Fig 21(b)). It has a preferred foliation for which every level $l$ (measured from some fiducial crown) contains $m$ elements, $\left\{e_{i}(l)\right\}$, where $i=1, \ldots m 10$ The level $l+1$ elements are related to the level $l$ elements as follows: $e_{i}(l+1) \succ e_{i}(l)$ and $e_{i+1}(l+1) \succ e_{i}(l)$. The transitive closure of this gives us the causal set. We will only use this preferred foliation for our choice of inextendible antichains, to make the discussion simple.

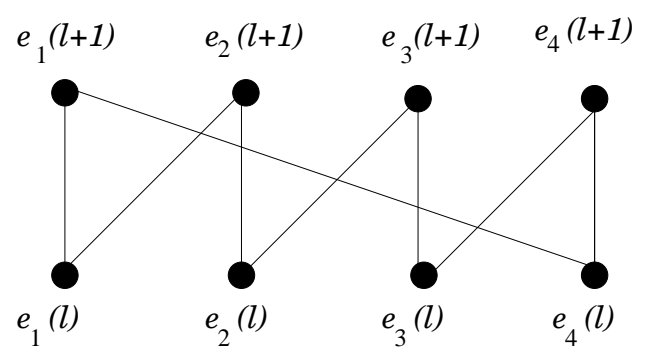

(a)

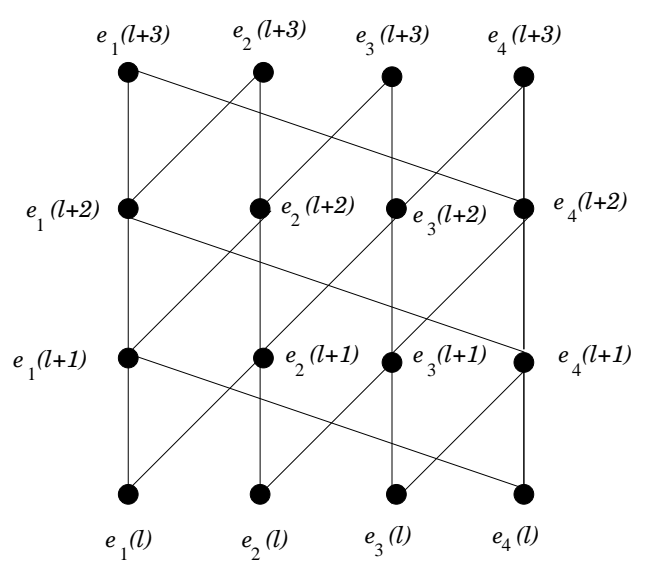

(b)

Figure 21: (a) An $m=4$ crown with 8 elements. (b) A tower of $m=4$ crowns.

Let us start with the $l=0$ level antichain $A$ and thicken to $T_{l}(A)$. Because of the regularity of the crown poset, this level thickening is closely related to volume

\footnotetext{
${ }^{10}$ The numeric labels used in the random antichain algorithm are ordered by layer, i.e. elements $0 \ldots m-1$ are in the bottom layer, $m \ldots 2 m-1$ in the second, etc.
} 
thickening. Thus, $l=1$ corresponds to a volume thickening $n=2$, level $l=2$ is $n=5, l=3$ to $n=9$. All intermediate values of $n$ are identical to the corresponding lower values. The set of shadows in $T_{1}(A)$ are then $\left\{S_{i}(1) \equiv\left\{e_{i}(0), e_{i-1}(0)\right\}\right\}$. $S_{i j}(1) \equiv S_{i}(1) \cap S_{j}(1) \neq \emptyset$ only for $j=i+1$ and $j=i-1$, with the intersections being $e_{i}(0)$ and $e_{i-1}(0)$ respectively. Thus, 3 -way and higher intersections vanish. For $m \geq 3$, the nerve $\mathcal{N}(1)$ associated with $T_{1}(A)$ is therefore a cycle of 1 -simplices, with $m$-vertices and $m$-edges as shown in Fig 22 (a). Thus $H_{0}=\mathbb{Z}$ and $H_{1}=\mathbb{Z}$, i.e. $\mathcal{N}(1)$ is homological to the circle. At level $l=2$, the shadows are $\left\{S_{i}(2) \equiv\right.$ $\left.\left\{e_{i}(0), e_{i-1}(0), e_{i-2}(0)\right\}\right\}$, and the largest intersections are 3 -way, $S_{i j k}(2) \equiv S_{i}(2) \cap$ $S_{j}(2) \cap S_{k}(2)$, with (i) $j=i+1, k=i+2, S_{i j k}(2)=e_{i}(0)$, (ii) $j=i+1, k=i-1$ $S_{i j k}(2)=e_{i-1}(0)$ or (iii) $j=i-1, k=i-2, S_{i j k}(2)=e_{i-2}(0)$. This gives a nerve simplicial complex $\mathcal{N}(2)$ made up of 2-simplices (Fig [22(b)), which for $m \geq 5$ is again homological to the circle. Note that for $m=4$, the non-zero homology groups are $H_{0}=\mathbb{Z}$, and $H_{2}=\mathbb{Z}$, which is homological to the sphere and not the circle.

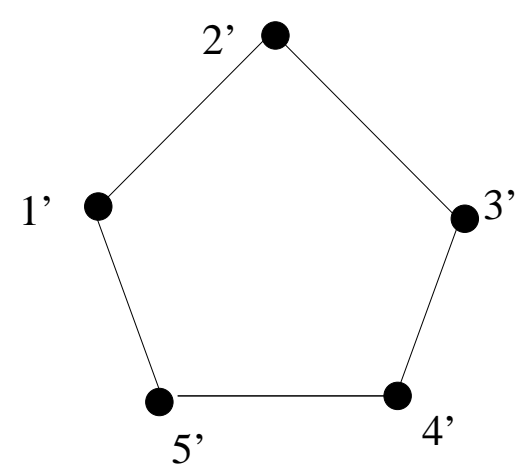

(a)

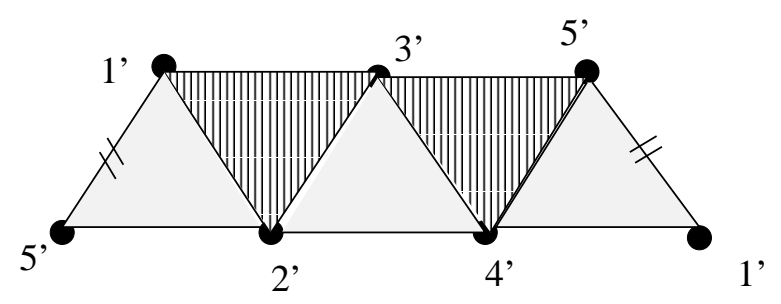

(b)

Figure 22: The nerves of (a) $T_{1}(A)$ and (b) $T_{2}(A)$ for $m=5$.

At level $l$, therefore, the shadows are $\left\{S_{i}(l) \equiv\left\{e_{i}(0), e_{i-1}(0), \ldots e_{i-l}(0)\right\}\right\}$, and one has non-trivial $l+1$ way intersections $S_{i_{1} i_{2} \ldots i_{l+1}}(l)$ for a consecutive set $\left\{i_{1}, i_{2} \ldots i_{l+1}\right\}$ thus giving rise to a higher dimension nerve simplicial complex. Now, as long as the shadows are small enough as in the example in Fig 22(b), these higher dimensional nerves are homological to the circle. Small enough is determined by taking recourse to the structure of $\mathcal{N}(1)$ which resembles a circle, for $m \geq 3$, and provides a nearest neighbour for every element in $A$. Let us make this more precise.

Let $N^{*}(1)$ be the dual complex of $\mathcal{N}(1)$ formed by taking vertices to edges and vice versa. The vertices of $N^{*}(1)$ are therefore the elements of $A$, and the edges, represent the level 1 cover of $A$, i.e. the elements of level 1 whose shadows cover $A$. Then there exists homotopy preserving maps $\Xi: N^{*}(1) \rightarrow S^{1}$ such that $p_{i}=\Xi\left(e_{i}(0)\right)$ are a distinct set of $m$ points on $S^{1}$ and the edges between $e_{i}(0)$ and $e_{i+1}(0)$ map to 
the connecting curve from $p_{i}$ to $p_{i+1}$ in $S^{1}$. One thus obtains from this a covering of $S^{1}$ by closed intervals. These can be extended trivially to open intervals, since $\Xi$ is only required to be homotopy preserving.

Consider the two shadows $S_{i}(l)$ and $S_{i-l}(l)$ whose intersection includes $e_{i-1}(0)$. If $i-2 l \bmod m \leq i \bmod m$, however, then $e_{i}(0)$ also belongs to the intersection. However, for $0<l<m, e_{i}(0)$ and $e_{i-1}(0)$ are not nearest neighbours on $N^{*}(1)$. Using the De Rham-Weil theorem [24] we see that the related covering of $S^{1}$ has intersections which are not themselves connected, and hence its nerve need not reproduce the homology of the circle. On the other hand, if $2 l<m$, all intersections are homotopically trivial, and so the nerve $\mathcal{N}(l)$ is homotopic to $S^{1}$.

Thus, for $m$ sufficiently large, one obtains a large range of thicknesses $1 \leq$ $l \leq[m / 2]$ for which the homology is stable. However, this stability sets in at the discreteness scale, unlike the case of manifoldlike causal sets, where $H_{0}$ rapidly decreases. If it were possible to isolate this class of antichains in the tower of crowns poset without any a priori knowledge of its structure, it would be clear that the causal set does not pass our test of manifoldlikeness.

However, our numerical test for manifoldlikeness uses random antichains and we do not look for the existence of special antichains from which to start the thickening. For a randomly chosen antichain it will generically be true that the homology changes for small $n$. Because of the crystalline structure, the number of inextendible antichains which have a distinct behaviour (i.e. cannot be mapped to each other via time or spatial translation) will depend on $m$ and hence doing a sufficiently large number of trials will uncover the repeated structure. It is relatively easy to see this for small $m$. The simplest case of $m=3$, for example, has only three distinct types of antichains, and so there are only 3 distinct possibilities for the homology as a function of $n$. As $m$ increases, the number of possible antichains becomes much larger, and so the test for repetition will need more trials.

We use the random antichain algorithm to run our test on $N=1000$ element tower of crowns causal sets with $m=3, m=4, m=10$ and $m=40$, and run the test up to the cosmological scale for the first three and $n_{m}=99$ for the last. For $m=3,4,10$, the cosmological scale is reached early and hence no stable homology is exhibited. Moreover, for small thickness, the homology groups are repeated in different trials as expected, so these causal sets clearly are not manifoldlike. Fig 23 shows the behaviour of $H_{0}$ at small thickness for 50 of the trials. Such perfect repetitions signal the fact that antichains which are identical, except for being time translated, appear more than once in the trials so that their homology groups are identical for all $n$.

For $m=40$, on the other hand, the set of 100 trials does not suffice to demonstrate that repetitions occur. Hence the behaviour for small $n$ appears to have the 


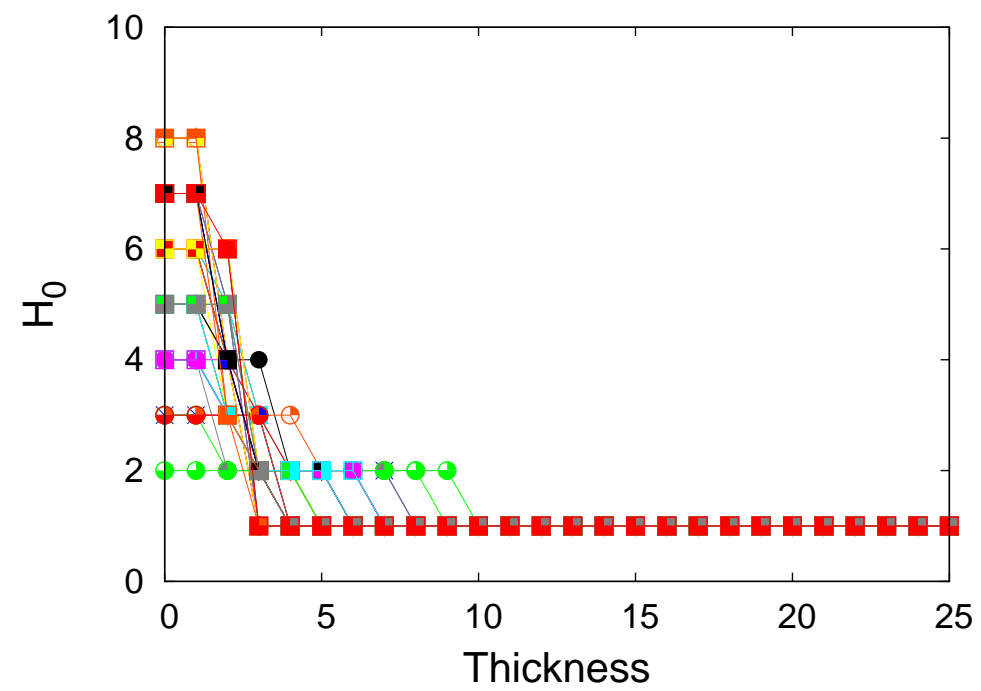

Figure 23: The fluctuations of the homology group $H_{0}$ for small $n$ for the $m=10$ tower of crowns in 50 trials are shown. Though not immediately clear from the figure, there is a repetition of the behaviour in a large number of the trials.

characteristics of the stochastic fluctuations of causal sets that are obtained from discretisations. Fig 24 shows the fluctuations in $H_{0}$ for 50 trials at small thickness. Moreover, the stability analysis shows that for all the 93 legitimate trials, the cylinder homology appears as the first stable region. Putting these ingredients together it seems that we can conclude that the $m=40$ tower of crown causal set satisfies our necessary but not sufficient test for manifoldlikeness when we restrict ourselves to 100 trials. Having passed this topological test, however it readily fails a crucial geometric test. The crystalline structure is immediately obvious when counting the valency or the number of links for each element - it is the same (and very small) for all 1000 elements, and this is a definite sign that the causal set is not manifoldlike. While geometric considerations rule out this simple example, it may not be as easy in general. Importantly, this example stresses that our test is only a sufficient condition and acts as a basic filter before using more subtle tests for manifoldlikeness.

We can use this example as a lesson that random antichains aren't always sufficient and that our tests should be made more sensitive. One could look for special classes of antichains to check if initial fluctuations in the homology exist, are generic or the homology versus thickening behaviour is the same for all the antichains. As shown analytically, the tower of crowns for any $m$ will not pass this test for antichains of cardinality $m$ which correspond to the level foliations. 


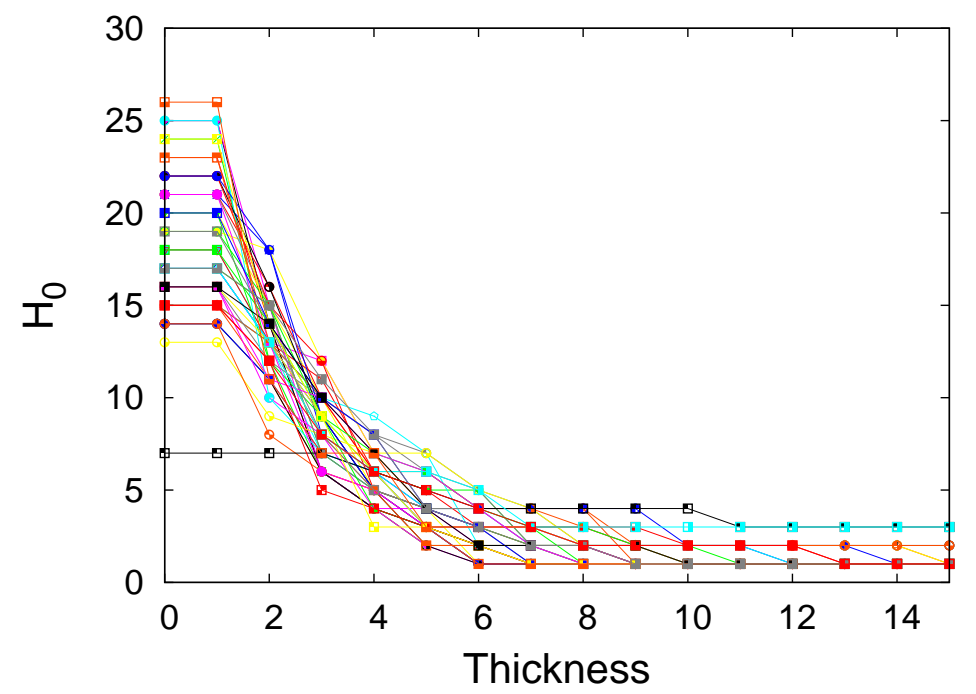

Figure 24: The fluctuations of the homology group $H_{0}$ for small $n$ for the $m=40$ tower of crowns in 50 trials are shown. This resembles more closely the fluctuations obtained from a causal set discretisation of the cylinder spacetime.

\section{Conclusion}

In this work we have presented evidence that manifoldlikeness in a causal set $C$ manifests itself in terms of a first stable homology for a large fraction of randomly chosen antichains, in a statistically large sampling of inextendible antichains. Moreover, for small thicknesses, the correlation between the homology across the different antichains is very weak, this reflecting the detail of the antichain itself, rather than being a robust feature of the continuum.

This hypothesis was reached by doing a large number of simulations for causal sets obtained via a sprinkling into a set of $2 \mathrm{~d}$ conformally flat spacetimes. It was then verified for causal sets obtained from sprinkling into a class of $3 \mathrm{~d}$ conformally flat spacetimes. However, the $3 \mathrm{~d}$ computations were computationally too intensive to allow any statistical analysis. Nevertheless, it is a worthwhile future exercise to demonstrate whether the above test for manifoldlikeness works for a wider class of examples in higher dimensions. It is also important to test how and when torsion makes its appearance. All our examples are simple enough topologically for it not to have made an appearance, and it would be useful to have a better understanding of this.

Our test for manifoldlikeness was then performed on examples of causal sets not obtained via sprinklings of continuum spacetimes. These were causal sets generated from a class of transitive percolation dynamics, and the regular tower of crowns 
causal sets. While the former class had stable regions, these were not consistent over a sufficient number of the trials and hence did not pass our test. However, the restriction to relatively large values of the parameter $p$ because of computational constraints should be borne in mind. This means that the causal sets are very narrow, and hence not likely to exhibit manifoldlike characteristics. Testing for manifoldlikeness in transitive percolation dynamics for smaller values of $p$ would therefore be worthwhile to study in the future.

The other class of causal sets studied, the tower of crowns, do pass the stability test, but the existence of special classes of antichains for which there is no fluctuating homology at small thicknesses means that they do not pass our test of manifoldlikeness.

We should also reiterate that our analytical understanding of the homology construction is limited to globally hyperbolic regions of spacetime. While it is true that there are large classes of spacetimes which are not globally hyperbolic, it is also true that for strongly causal spacetimes, there are convex normal neighbourhoods (CNNs) around every point which are themselves globally hyperbolic. Our algorithm currently does not pick such neighbourhoods, but considers the global topology of the spacetime. Ultimately, we will also need to include checks for CNNs, for which the topology should be trivial. For this, however, we would need to understand better how geometry is encoded in a causal set.

If this test for manifoldlikeness is robust, and survives further analysis, it would provide us a valuable tool in assessing manifoldlikeness in causal sets generated by causal set quantum gravity. Conversely, it is possible that these results can suggest a means of incorporating the right sort of locality into the quantum dynamics [32] so that manifoldlike causal sets emerge in the classical limit of the theory.

Acknowledgements: We thank Rafael Sorkin, Sanjib Sabhapandit and Dan Christensen for useful discussions. We are extremely grateful to Pawel Pilarczyk for providing code to simplify our simplicial complexes, and for numerous suggestions on how to use his homology software (CHomP). This work was supported in part by the Royal Society International Joint Project 2006-R2 and the computations were carried out using the Raman Research Institute high performance computers, and the Shared Hierarchical Academic Research Computing Network (SHARCNET:www.sharcnet.ca). This work was also supported in part by the Perimeter Institute for Theoretical Physics. Research at Perimeter Institute is supported by the Government of Canada through Industry Canada and by the Province of Ontario through the Ministry of Research \& Innovation. DR was supported in part by the Marie Curie Research and Training Network ENRAGE (MRTN-CT-2004-005616). 


\section{References}

[1] David Malament, "The class of continuous timelike curves determines the topology of space-time", J. Math. Phys 181399 (1977).

[2] L. Bombelli, J. H. Lee, D. Meyer and R. Sorkin, "Space-Time As A Causal Set," Phys. Rev. Lett. 59, 521 (1987).

[3] R. D. Sorkin, School on Quantum Gravity, Valdivia, Chile, 4-14 Jan 2002. Published in Valdivia 2002, "Lectures on quantum gravity" 305-327, arXiv:gr-qc/0309009.

[4] Joe Henson. The causal set approach to quantum gravity. In D. Oriti, editor, Approaches to Quantum Gravity - Towards a new understanding of space and time. Cambridge University Press, 2006. gr-qc/0601121.

[5] David P. Rideout and Rafael D. Sorkin. "Classical sequential growth dynamics for causal sets." Phys. Rev. D, 61:024002, 2000. gr-qc/9904062.

[6] R. D. Sorkin, "Quantum Dynamics without the Wave Function," J. Phys. A 40, 3207 (2007) arXiv:quant-ph/0610204.

[7] R. Sverdlov and L. Bombelli, "Gravity and Matter in Causal Set Theory," arXiv:0801.0240 [gr-qc].

[8] G. Brightwell, J. Henson and S. Surya, "A 2D model of Causal Set Quantum Gravity: The emergence of the continuum," Class. Quant. Grav. 25, 105025 (2008) arXiv:0706.0375 [gr-qc]].

[9] L. Bombelli, J. Henson and R. D. Sorkin, "Discreteness without symmetry breaking: A theorem," arXiv:gr-qc/0605006.

[10] D.A. Meyer, The Dimension of Causal Sets. Ph.D. thesis, MIT (1988).

[11] G. Brightwell and R. Gregory, "The Structure Of Random Discrete SpaceTime," Phys. Rev. Lett. 66, 260 (1991).

[12] D. Rideout and P. Wallden, "Emergent Continuum Spacetime from a Random, Discrete, Partial Order," arXiv:0811.1178 [gr-qc]; D. Rideout and P. Wallden, "Spacelike distance from discrete causal order," arXiv:0810.1768 [gr-qc].

[13] Seth Major, David Rideout, and Sumati Surya. "On recovering continuum topology from a causal set." J. Math. Phys., 48, 032501, 2007. gr-qc/0009063. 
[14] Seth Major, David Rideout, and Sumati Surya. "Spatial hypersurfaces in causal set cosmology." Class. Quantum Grav., 23:4743-4751, 2006. grqc/0506133.

[15] T. Goodale, G. Allen, G. Lanfermann, J. Massó, T. Radke, E. Seidel, and J. Shalf, "The Cactus framework and toolkit: Design and applications," in Vector and Parallel Processing - VECPAR 2002, 5th International Conference, pp. 197-227. Springer, Berlin, 2003.

[16] The causal set toolkit is described briefly in:

D. Rideout and P. Wallden, "Spacelike distance from discrete causal order," arXiv:0810.1768 [gr-qc], and D. Rideout and S. Zohren, "Evidence for an entropy bound from fundamentally discrete gravity," Class. Quant. Grav. 23, 6195 (2006) [arXiv:gr-qc/0606065].

[17] Pawel Pilarczyk et. al. Chomp. http://chomp.rutgers.edu.

[18] X. Martin, D. O'Connor, D. P. Rideout and R. D. Sorkin, "On the 'renormalization' transformations induced by cycles of expansion and contraction in causal set cosmology," Phys. Rev. D 63, 084026 (2001) arXiv:gr-qc/0009063].

[19] A. Ash and P. McDonald, J. Math. Phys. 44, 1666 (2003) arXiv:gr-qc/0209020.

[20] A. Ash and P. McDonald, J. Math. Phys. 46, 062502 (2005).

[21] Joe Henson. "Constructing an interval of Minkowski space from a causal set." Class.Quant.Grav., 23:L29-L35, 2006. gq-qc/0601069.

[22] Grisha Perelman, The entropy formula for the Ricci flow and its geometric applications, arXiv .org:math/0211159.

[23] James R. Munkres. Elements of Algebraic Topology. Addison-Wesley, 1984.

[24] A. Weil, "Sur les theoremes de de Rham", Commentarii Mathematici Helvetici 26, 119-145 (1952); Georges De Rham, "Complexes automorphismes et homomorphie diffrentiable", Annales de l'institut Fourier, 2 51-67 (1950).

[25] R.P. Stanley, Enumerative Combinatorics, vol. 1, Wadsworth and Brooks/Cole, Pacific Grove, CA, 1986 G/A second printing, Cambridge University Press, Cambridge, 1997;

[26] S. Surya, "Causal Set Topology," Theor. Comput. Sci. 405, 188 (2008) arXiv:0712.1648 [gr-qc]]. 
[27] R. D. Sorkin, "Does locality fail at intermediate length scales?", in D. Oriti (ed), "Approaches to Quantum Gravity - Toward a new understanding of space and time", Cambridge University Press, 2006; J. Henson, "The causal set approach to quantum gravity," in D. Oriti (ed), "Approaches to Quantum Gravity - Toward a new understanding of space and time", Cambridge University Press, 2006.

[28] R. P. Geroch, "Topology in general relativity," J. Math. Phys. 8, 782 (1967).

[29] R. D. Sorkin, "On Topology Change And Monopole Creation," Phys. Rev. D 33, 978 (1986).

[30] F. Dowker and S. Surya, Phys. Rev. D 58, 124019 (1998) arXiv:gr-qc/9711070.

[31] M. Varadarajan and D. Rideout, "A general solution for classical sequential growth dynamics of causal sets," Phys. Rev. D 73, 104021 (2006) arXiv:gr-qc/0504066.

[32] J. Henson, "Comparing causality principles," Stud. Hist. Philos. Mod. Phys. 36, 519 (2005) arXiv:quant-ph/0410051. 